\title{
Multiple Excitation in Photoionization Using B-splines
}

\author{
T. N. Chang ${ }^{a}$ and T. K. Fang ${ }^{b}$ \\ ${ }^{a}$ Department of Physics and Astronomy, University of Southern California, Los \\ Angeles, CA 90089-0484, U.S.A. \\ ${ }^{b}$ Department of Physics, Fu Jen Catholic University, Taipei, Taiwan 242, ROC
}

\begin{abstract}
A bstract
We present a review of a few recent applications of three B-spline based methods to atomic photoionization dominated by multiple excitation for both single and multiple ionization channels. The emphases are given to physical interpretation of atomic processes based on the energy variation of effective one-particle orbital functions and their corresponding scattering phase shifts or eigenphase shifts extracted from the state wavefunctions. A number of general features which characterize the photoionization resonance spectra and a few exceptions due to interference between overlapping autoionization series are given.
\end{abstract}

Keywords: Photoionization; B-splines; Multiple excitation; configuration interaction; K-matrix; Complex-rotation

PACS: 32.80.Fb; 32.80.Dz; 32.70.Jz 


\section{Introduction}

Shortly after the successful quantitative demonstration of the application of B-splines by Johnson et al (1988) in a relativistic many-body perturbation theory (MBPT) calculation, Chang (1989a, 1989b) had shown that the B-spline-based orbitals can also be employed effectively in nonrelativistic configuration-interaction calculations. Froese Fischer and her co-workers (Froese Fischer and Idrees, 1990; Brage et al, 1992; Froese Fischer, 1993) had independently applied the B-splines to the continuum with success. Other earlier works employing nonrelativistic B-spline-based orbitals include calculations by Chang (1990, 1993a), Tang et al (1990), Chang and Wang (1991a, 1991b), Chang and Tang (1991, 1992), Chen and Hsue (1992), Chen (1993), van der Hart and Hansen (1992a, 1992b, 1993), Chang and Zhen (1993), Chang and Zhu (1993), Tang and Bachau (1993), Cormier et al (1993), Xi et al (1993), and Decleva et al (1994). Extension to the relativistic configuration-interaction procedure was also carried out by Chen et al (1993).

Other existing theoretical approaches for multiple excitations in continuum include the multichannel quantum defect theory (MQDT) with empirical mixing parameters. MQDT is perhaps one of the most efficient approaches in the systematic interpretation of interaction between two or more interacting excitation channels (Seaton, 1966; Lu and Fano, 1970; Lu, 1971; Lee and Lu, 1973). An ab initio MQDT application requires an accurate quantitative determination of the MQDT parameters from the low to intermediate excited states which are strongly affected by short-range multi-electron interaction. Early attempts using the close-coupling approach (Dubau and Wells, 1973; Thompson et al, 1974; Norcross and Seaton, 1976; Mendoza, 1981) had led to varying degrees of success. The extension of the eigenchannel $\mathrm{R}$-matrix method has provided a direct calculational procedure for the determination of the MQDT mixing parameters (Greene and Kim, 1987; Aymer et al, 1996; Pan et al, 1996). Extensive applications of the eigenchannel R-matrix method (Robicheaux and Greene, 1993a, 1993b; Wood and Greene, 1994) to the study of the autoionization series in heavy metal atoms were reported with success. Efforts were also made to include the effect of radiative damping when the non-radiative autoionization is comparable to the radiative decay (Bell and Seaton, 1985; Robicheaux et al, 1995). An alternative and more straightforward $\mathrm{R}$-matrix calculational procedure was developed by Burke and his co-workers (Burke, 1993). Its ability to provide reliable atomic structure calculation were demonstrated using the R-matrix with pseudostates method in a few earlier applications (Bartschat et al, 1996; Hudson et al, 1996). Following the adiabatic hyperspherical coordinate method developed by Macek (1968) and Lin (1976, 1984, 1986), Sadeghpour and Greene (1990) derived a simple expression which yields the positions of dominant resonances for $\mathrm{H}^{-}$. An adiabatic hyperspherical approach was proposed by Starace and his co-workers to study the multi-photon detachment processes in two-electron systems (Liu et al, 1992; $\mathrm{Du}$ et al, 1993). Also, a close coupling hyperspherical method was formulated to study the doubly excited resonances in two-electron systems by Tang et al $(1992,1993)$, Zhou and Lin (1994) and Tang and Shimamura (1995).

In addition, Froese Fischer and her co-workers extended the multiconfiguration HartreeFock (MCHF) approach to include the continuum wave function corresponding to the ionization channel at energies near the doubly excited resonances in their photoionization study of the ${ }^{1} \mathrm{P}$ autoionization series of $\mathrm{Mg}$ (Froese Fisher and Saha, 1987) and the 
autoionization rate of three-electron systems (Miecznik et al, 1993). Other MCHF applications to atomic structure calculation include the works by Vaeck et al (1991) and by Kim et al (1997a, 1997b). Altun and Manson (1996) extended the MBPT approach to photoionization from excited atoms. Alternatively, Moccia and Spizzo (1987, 1990, and 1991) successfully developed an $L^{2}$ calculational procedure to represent the continuum spectrum of two-electron and divalent atoms by using a set of elaborate basis functions including a modified Slater-type-orbital with an explicit $\cos (k r)$ dependence. Other $\mathrm{L}^{2}$ approach for studying of multiple excitations includes the saddle-point complex-rotation method by Chung and Guo (1995).

Although the detailed calculational procedures may differ in these theoretical approaches, the success of all these approaches depends largely on their ability to take into account the strong short-range multi-electron interactions. In this paper, we will review a few B-spline based methods and their applications to atomic photoionization dominated by double excitations. What make the B-spline based approach attractive are a few important features. First, with an equal amplitude for all B-splines uniformly spread over the entire space between $r=0$ and $r=R$, it offers a unified approach which is equally effective in studying the discrete states (including the highly excited bound states) and continuum spectra. The bound-bound and bound-continuum transition matrices can be evaluated at the same time within a single calculation. Second, the use of a pre-determined quasi-complete set of one-particle orbitals eliminates the need of any a priori procedure in selecting the parameters for the exponential functions required in many other $L^{2}$ based CI (configuration interaction) calculations which often depend upon non-trivial treatment to minimize the linear dependence embedded in the choice of basis functions. In our B-spline based approach, the transition matrix can be evaluated without the presence of non-vanishing overlap between orbital functions due to the rigorous orthogonality (often up to $10^{-12}$ or better) between one-electron radial functions. Although this choice of basis functions requires intensive computationally effort, it nevertheless simplifies tremendously the numerical algorithm. Consequently, it is much easier to take advantage of the parallel computing environment which has become increasingly easy to manage. Third, the individual contribution from multi-electron interaction corresponding to different configuration combination can be conveniently identified and estimated. For transitions dominated by the double excitations, the resonant structures can be linked directly to the excitations of individual electrons. Also, the energy variation of the effective orbital functions $\xi_{n l}$ of the excited or ionized electron, defined by Eq. (10) of Chang and Tang (1991) or Eq. (50) of Chang (1993b), can be extracted explicitly from the massive CI calculation. The widths of the resonances are determined from the calculated energy variation of the scattering phase shifts for a single ionization channel and the total eigenphase shifts for multiple ionization channels. As a result, it can easily lead to detailed physical interpretation of the transition processes.

In section 2, we will review briefly three B-spline based theoretical methods: i) BSCI (B-spline based configuration interaction) method for single channel photoionization, ii) BSK (B-spline based K-matrix) method for multi-channel photoionization, and iii) BSCR (B-spline based complex-rotation) method, also for multi-channel ionization. A number of applications of these three methods will be discussed in section 3 . In section 4 we will comment on the future direction and the works currently in progress. 


\section{Theory}

All three B-spline based methods discussed in this paper starts with a quasi-complete set of discretized one-particle functions $\chi_{n l}(r)$ corresponding to an electronic orbital $n l$ with an orbital angular momentum $l$ but variable energy (both negative and positive) defined by an effective one particle hamiltonian $h_{l}^{\text {eff }}$, i.e.,

$$
h_{l}^{e f f} \chi_{n l}=\epsilon_{n l} \chi_{n l}
$$

with a boundary condition that $\chi_{n l}(r=R)=0$. A set of $N$ B-splines $B_{i, K}(r)$ of order $K$ (de Boor, 1978) is defined inside a sphere of radius $R$. In all three B-spline based methods discussed in this section, the solution $\chi_{n l}$ is expanded as

$$
\chi(r)={ }_{i}^{\chi^{N}} C_{i} B_{i}(r)
$$

where the index $K$ is dropped for simplicity.

The Hamiltonian matrix is typically constructed with J-dependent basis function $\psi_{n_{1} l_{1}, n_{2} l_{2}, \cdots}^{\Omega}\left(\vec{r}_{1}, \vec{r}_{2}, \cdots\right)$, which is characterized by an electronic configuration $\left(n_{1} l_{1}, n_{2} l_{2}, \cdots\right)$ and a set of quantum numbers $\Omega \equiv\left(S L J M_{J}\right)$, where $S, L, J$, and $M_{J}$ are the total spin, the total orbital angular momentum, the total angular momentum and its corresponding magnetic quantum number, respectively. The basis function $\psi_{n_{1} l_{1}, n_{2} l_{2}, \ldots}^{\Omega}$ represents a sum of J -independent basis function $\psi_{n_{1} l_{1}, n_{2} l_{2}, \ldots}^{\Lambda}$ over all $M_{S}$ and $M$, where $\Lambda \equiv\left(S L M_{S} M\right)$ is a set of quantum numbers $S, L, M_{S}$, and $M . M_{S}$ and $M$ are the magnetic quantum numbers of $S$ and $L$, respectively. Within the central field approximation, the basis function $\psi_{n_{1} l_{1}, n_{2} l_{2}, \ldots}^{\Lambda}$ is expressed as a sum of n-particle Slater determinant wave functions corresponding to the configuration $\left(n_{1} l_{1}, n_{2} l_{2}, \cdots\right)$ over all magnetic quantum numbers with an appropriate angular momentum coupling. The Slater determinant wave functions are constructed in the usual form as the product of one-particle orbitals with its radial function given by Eq. (1). A more detailed discussion of $\chi(r)$ is given elsewhere (Chang and Tang, 1991; Chang, 1993b; Fang and Chang, 2000).

\subsection{B-spline based configuration interaction (BSCI) method}

The applications of the BSCI method in the past decade have offered a number of interesting physical interpretations of the structure profiles dominated by multi-electron interaction in single channel photoionization for two-electron and divalent atoms. The detailed theory was first developed by Chang and Tang (1991) and subsequently reviewed by Chang (1993b). The photoionization cross section $\sigma$ is given by

$$
\sigma=\frac{4 \pi \alpha}{k A^{2}} f_{E I}
$$


where $k$ is the momentum of the ionized outgoing electron, $\alpha$ is the fine-structure constant, and $f_{E I}$ is an effective oscillator strength for transition from the initial to final state detailed elsewhere (Chang, 1987; and section 2.4 in Chang, 1993b). The amplitude $A$ and the scattering phase shift $\delta_{\ell}$ are evaluated by fitting the calculated effective one-particle orbital function $\xi_{\epsilon \ell}(r)$ to its corresponding asymptotic expression at large $r$, i.e.,

$$
\xi_{\epsilon \ell}(r) \longrightarrow A\left[\frac{k}{\zeta(r)}\right]^{\frac{1}{2}} \sin \left[\phi(r)+\delta_{\ell}\right]
$$

As $r \rightarrow \infty, \zeta \rightarrow k$ and

$$
\phi \longrightarrow\left(k r+\frac{Q}{k} \ln (2 k r)-\frac{\ell \pi}{2}+\delta_{C}\right)
$$

where $Q$ is the effective nuclear charge experienced by the outgoing electron and $\delta_{C}$ is the Coulomb phase shift. At an energy close to a doubly excited resonance, the scattering phase shift $\delta_{\ell}(E)$ increases rapidly by a total of $\pi$ and follows an expression given by

$$
\delta_{\ell}(E)={ }_{i=0}^{X^{2}} a_{i} E^{i}+\tan ^{-1} \frac{\Gamma / 2}{E_{r}-E} .
$$

The resonance energy $E_{r}$ and the width $\Gamma$ are determined by a least-square fit of the calculated energy varying $\delta_{\ell}(E)$ to Eq. (6). Detailed computational procedures are given in Chang (1993b).

The numerical works required in a BSCI calculation consist of four key steps. The first step solves a set of one-particle integral differential equations with non-local terms (such as a Hartree-Fock equation). The computer code was developed using the procedure detailed in Chang (1993b), which requires little CPU memory or time. The second step evaluates a hamiltonian matrix of a size often up to 10,000 or greater. The value of the individual matrix element, which represents a two-variable integral over a two-body Coulomb interaction is in general small but non-zero and, as a result, the matrix is non-sparse. The calculation is performed by dividing the matrix into a large number of sub-matrices of a size of 100 to 200 each. On a single-node CPU, these sub-matrices are calculated sequentially, although, in principle, they are independent of each other and can be evaluated individually. The extension to parallel computing environment speeds up this step substantially. The third step involves the calculation of a limited number of energy eigenvalues and their corresponding eigenvectors by diagonalizing the non-sparse matrix generated in the second step. A direct diagonalization using the standard code on a single-node machine requires a RAM approximately two to four times the size of the matrix, or, 2-4 GB RAM or more in our BSCI applications. This memory requirement exceeds a typical 1-2 GB RAM available on a standard single-node CPU. To circumvent this obstacle, a two-step diagonalization procedure with a memory requirement about half the size of a real and symmetric non-sparse matrix was developed. Details of this procedure are given elsewhere (Chang, 1993b). Although the memory requirement is substantially reduced, substantial RAM is still needed for a typical calculation. In general, the parallel computing environment does not offer substantial advantage for matrix 
diagonalization. However, one of the distinct features of the two-step diagonalization procedure is its ability to evaluate each set of eigenvalue and its corresponding eigenvector individually. In other words, it offers a very attractive computational alternative. The matrix can be stored distributively on each of the CPU in a multi-node cluster to meet the memory requirement. The evaluation of each set of eigenvalue and its corresponding eigenvector can be performed individually on each CPU while the input matrix is shared by using the standard MPI (message passing interface) technology in parallel computing environment. The fourth step involves the calculation of the transition amplitude and the cross section. It can be performed on any single-node CPU.

\subsection{B-spline based multichannel K-matrix (BSK) method}

For multi-channel photoionization, Fang and Chang (2000) has successfully extended the BSCI method following the usual K-matrix approaches (Starace, 1980; Carravetta et al, 1993). An eigenchannel, designated by a channel index $\Gamma$ at a total energy $E$, is given as a linear combination of individual open channel wavefunctions $\left|\Phi_{\gamma_{0} E}\right\rangle$, i.e.,

$$
|\Gamma E\rangle={ }_{\gamma_{0}}^{\mathrm{X}}\left|\Phi_{\gamma_{0} E}\right\rangle U_{\gamma_{0} \Gamma}(E) \cos \eta_{\Gamma}(E)
$$

where $U_{\gamma_{0} \Gamma}(E)$ is an orthogonal transformation matrix at an energy $E$ and the eigenphase shift $\eta_{\Gamma}$ represents the interactions between $\left|\Phi_{\gamma_{0} E}\right\rangle$. Similar to the normal modes of a complex system, the eigenchannels are intimately related to the dynamics of the atomic process, although they do not in general represent directly the individual atomic states.

The eigenstate $|\Gamma E\rangle$ satisfies the orthonormality relation

$$
\left\langle\Gamma^{\prime} E^{\prime} \mid \Gamma E\right\rangle=\delta_{\Gamma^{0} \Gamma} \delta\left(E^{\prime}-E\right) .
$$

The transformation matrix $U_{\gamma_{0} \Gamma}(E)$ and the eigenphase shift $\eta_{\Gamma}$ are obtained by diagonalizing the on the energy shell $K$-matrix, i.e.,

$$
{ }_{\gamma}\left\langle\gamma_{o} E|K(E)| \gamma E\right\rangle U_{\gamma \Gamma}=-\pi^{-1} \tan \eta_{\Gamma} U_{\gamma_{0} \Gamma}
$$

where the K-matrix, expressed as a set of coupled integral equations, is given explicitly by Eq. (11) in Fang and Chang (2000). Similar to the increase of the scattering phase shift by a value of $\pi$ across a resonance embedded in a single continuum, the sum of the eigenphase shift over all eigenchannels, i.e.,

$$
\eta_{t o t}=\stackrel{X}{\eta_{\gamma}}
$$

also increases by a total of $\pi$ as the energy increases across an isolated resonance. As a result, the total width of a resonance can be calculated from the energy variation of $\eta_{t o t}$. 
Following the detailed procedure outlined in Fang and Chang (2000), the total photoionization cross section $\sigma_{t o t}$ is given by the sum of the partial cross sections over all open channels, i.e.,

$$
\sigma_{t o t}={ }_{\gamma}^{\mathrm{X}} \sigma_{\gamma} .
$$

The partial cross section in unit of $a_{0}^{2}$ for each open channel $\gamma$ is given by

$$
\sigma_{\gamma}=4 \pi^{2} \alpha f_{\gamma I}
$$

where the effective oscillator strengths $f_{\gamma I}$ from an initial state I, corresponding to length and velocity approximations are given by Eqs. (22) and (23) in Fang and Chang (2000), respectively.

\subsection{B-spline based complex-rotation (BSCR) method}

The complex-rotation method offers an attractive alternative in the calculation of the resonance energies and widths for atomic resonances. By using a complex scaled hamiltonian (Ho, 1983) or a set of complex basis functions (Rescigno et al, 1978; Rescigno and McCurdy, 1985), the problem of finding resonance states is reduced to the solution of a complex eigenvalue problem, where the technique of bound-state configuration interaction can be used. Recently, the complex-rotation method has also been applied to the study of doubly excited resonances in the presence of an external electric or magnetic field (Fang and Ho, 1999).

The first application using complex basis functions to photoionization in a multielectron atom was carried out by Rescigno (1985). Instead of a complex scaled hamiltonian, B-spline-based complex basis functions are used in BSCR method to circumvent the difficulties encountered on the rotation of the radial coordinates in the hamiltonian (Fang et al, 2002). In the radial function of the outer electron in each open channel, the radius $r$ is replaced by $r \rightarrow r e^{-i \theta}$, or,

$$
\chi\left(r e^{-i \theta}\right)={ }_{i=1}^{X^{N}} C_{i} B_{i}\left(r e^{-i \theta}\right),
$$

whereas for the closed channels, the Hamiltonian remains real. At large $r$, numerically, the complex radial function $\chi$ diverges as a hyperbolic function. This difficulty is removed by the introduction of an exponential decay factor $\beta$ in a modified complex radial function, i.e.,

$$
\tilde{\chi}\left(r e^{-i \theta}\right)={ }_{i=1}^{X^{N}} C_{i} \tilde{B}_{i}\left(r e^{-i \theta}\right),
$$


where $\tilde{B}_{i}=B_{i}(r) e^{-\beta r}$. Following the usual complex rotational method, one first constructs the complex hamiltonian which is readily solved for the complex eigenstates $\Psi_{\nu}(\theta)$ and the complex eigenvalues $E_{\nu}(\theta)$. The photoionization cross section from an initial state $\Phi_{0}$ with energy $E_{0}$ is given by (Rescigno, 1985)

$$
\sigma(E)=4 \pi \alpha(\Delta E)^{\omega} \operatorname{Im}\left({ }_{\nu}^{\mathrm{X}} \frac{\left\langle\Phi_{0}|D| \Psi_{\nu}(\theta)\right\rangle^{2}}{E_{\nu}(\theta)-E}\right),
$$

where $\Delta E=E-E_{0}$ is the transition energy. In the length approximation, the dipole operator $D=\hat{\epsilon} \cdot\left(\vec{r}_{1}+\vec{r}_{2}\right)$ and $\omega=1$ and in the velocity approximation, $D=\hat{\epsilon} \cdot\left(\vec{\nabla}_{1}+\vec{\nabla}_{2}\right)$ and $\omega=-1$, where $\hat{\epsilon}$ represents the light polarization.

The exponential decay factor $\beta$ is determined by the condition

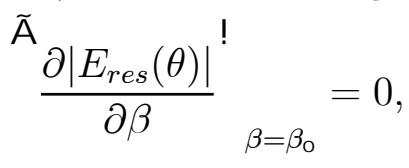

where $E_{\text {res }}(\theta)=E_{r}-i \Gamma / 2$ is the complex resonance energy and $\Gamma$ is the corresponding resonance width. The success of the BSCR method depends largely on the stability of the value $\beta_{o}$ as $\theta$ varies. One of the key advantages of the BSCR method is its ability to achieve the same numerical accuracy as the BSK method with a substantially smaller size in basis functions for the hamiltonian matrix. More details of the BSCR method are outlined in Fang et al (2002).

\section{A pplications}

The single channel photoionization dominated by an isolated doubly excited resonance may be conveniently described by the Fano formula (Fano, 1961) in terms of an asymmetry parameter $q$ and the smoothly varying background cross section $\sigma_{b}$, i.e.,

$$
\sigma(E)=\sigma_{b} \frac{(q+\epsilon)^{2}}{1+\epsilon^{2}}
$$

where $\epsilon=\left(E-E_{r}\right) /\left(\frac{1}{2} \Gamma\right)$ is the reduced energy defined in terms of the energy $E_{r}$ and the width $\Gamma$ of the resonance. The cross section $\sigma$ is expected to reach its peak value $\sigma_{\max }=\sigma_{b}\left(1+q^{2}\right)$ and a zero at energies

$$
E_{\max }=E_{r}+\frac{1}{2}(\Gamma / q) \text { and } E_{\min }=E_{r}-\frac{1}{2}(\Gamma q)
$$

respectively. The asymmetry parameter $q$ measures qualitatively the interference between transitions from initial state to final bound and continuum components of the state wavefunction. If the contribution for transition from the initial state to the bound component (i.e., bound-to-bound, or B-B transition) of the resonant state is very small in 
comparison with the one for the transition to the continuum background (i.e., bound-tocontinuum, or B-C transition), $q$ is very small and a zero cross section is expected either at or near $E_{r}$. The structure profile for such a resonance is often known as a window resonance. In contrast, if the photoionization is strongly dominated by the B-B transition, the $q$ value is large, and a more symmetric resonance structure is expected. For an intermediate $q$ value, the contributions from B-B and B-C transitions are comparable and the resonant profile is generally asymmetric. Unlike the $q$ parameter, the resonant width $\Gamma$, which measures the interaction strength between the bound and continuum components of the state wavefunction and in turn, determines the nonradiative decay rate of a resonance through autoionization, is independent of the transition process.

Except for a few examples to be discussed in section 3.2, the single channel ground state photoionization, in general, exhibits similar structure profile along the same au-

toionization series. For a two-electron atom or a light alkali-earth atom, as the effective principal quantum number $\nu$ increases, the resonance width $\Gamma$ decreases as $\nu^{-3}$. For higher angular momentum states, the resonance widths could be very small and the average lifetime due to autoionization for the doubly excited resonance could be comparable or longer than the radiative process. In other words, the decay of the resonance may be dominated by the radiative process similar to a bound excited state, instead of the usual autoionization.

For multi-channel photoionization, due to the presence of multiple ionization paths, the decay of the resonance is always non-radiative and dominated by the autoionization. In addition, the structure profile may change substantially along the same autoionization series due to the interaction between overlapping autoionization series. In fact, the overlap between resonances could be nearly complete and, without the detailed analysis based on theoretical calculation, it is virtually impossible to resolve such overlap experimentally. In this section, we will present a number of general features and exceptions based on detailed calculations using the B-spline based methods reviewed in section 2 .

\subsection{W indow resonances}

The near-symmetric structure profile of a window resonance can often be attributed directly to the near-zero contribution from the B-B transition. In general, the transition amplitude due to the B-C transition as a function of energy changes sign near the resonance energy $E_{r}$ as the effective one-electron orbital function for the outgoing ionized electron changes sign due to the rapid increase of the scattering phase shift by a total of $\pi$ across the resonance. A text book example can be found in the ground state photoionization of Be atom or Be-like ions to the $2 p n(\geq 3) s^{1} P$ resonant series (Chang and $\mathrm{Zhu}, 1995)$. Fig. 1 presents the energy variation of the effective oscillating orbital functions $\xi_{\epsilon p}(r)$ across the $2 p 5 s^{1} P$ resonance of Be-like $C$ III near the resonance energy at $\epsilon=k^{2}=0.17989 \mathrm{Ry}$. The scattering phase shifts $\delta_{p}$ are also given. The dominant $\mathrm{B}-\mathrm{C}$ contribution to the transition amplitude can be estimated by the corresponding $2 s \rightarrow \epsilon p$ one-electron dipole matrix elements $<\xi_{\epsilon p}|r| \xi_{2 s}>$ as a function of energy shown in Fig. 2. The contribution from the B-B transitions is small in comparison. This can be understood by considering the composition of the ground state of Be-like atom, which is approximately represented by a mixture of a dominating $2 s^{2}$ configuration and a small mixture of $2 p^{2}$ configuration. Two processes are expected to contribute 
to the B-B transition amplitude. First, the contribution from $2 s^{2} \rightarrow 2 p n(\geq 3) s$ is generally very small due to the simultaneous change of electron orbitals of two electrons. Second, the transition from the small $2 p^{2}$ mixture to $2 p n(\geq 3) s$ corresponds essentially to a $2 p \rightarrow n(\geq 3) s$ one-electron process and its near zero transition amplitude is expected from the very small oscillator strength known for the $2 s 2 p^{1} P \rightarrow 2 s n(\geq 3) s^{1} S$ transition for $\mathrm{Be}$ and Be-like ions (Chang and $\mathrm{Mu}, 1990)$. Consequently, the nearsymmetric photoionization structure profile for a typical window resonance results from two qualitative features involving the dipole matrix elements $<\xi_{\epsilon p}|r| \xi_{2 s}>$. First, the photoionization is dominated by the $2 s \rightarrow \epsilon p$ B-C transition. Second, a near-constant amplitude $A$ of the oscillating orbital functions away from the resonance energy $E_{r}$ shown in Fig. 1 is responsible for the near-symmetric dipole matrix elements shown in Fig. 2, and it ultimatically leads to the near-constant cross sections on both sides of the resonance.

We have also identified in our BSCI photoionization calculation a narrow $3 p 6 g^{1} F$ window resonance for $\mathrm{Al}^{+}$from bound excited $3 s \nu_{i} d^{1} D$ state, which, in contrast, is dominated by the B-B transition and radically different in nature from the one discussed above. This narrow $3 p 6 g^{1} F$ resonance shown in Fig. 3 is located on the higher-energy shoulder of its corresponding broad $3 s 6 d^{1} D \rightarrow 3 p 6 d^{1} F$ resonance dominated by the $3 s \nu_{i} d^{1} D \rightarrow 3 p \nu d^{1} F$ shakeup process involving the outer $d$ electron following the $3 s \rightarrow$ $3 p$ core electron excitation (Fang et al, 1997). Qualitatively, the $3 s \nu_{i} d^{1} D$ initial state interacts strongly with the $3 p p$ and $3 p f$ configuration series and the $3 s \epsilon f^{1} F$ continuum mixes substantially with the $3 p d$ and $3 p g$ configuration series. Below the $1 s^{2} 2 s^{2} 2 p^{6} 3 p^{2} P$ threshold, the photoionization spectra feature two autoionization series, i.e., a broad $3 p n d^{1} F$ and a narrow $3 p n g{ }^{1} F$ series. The contributions to the total transition amplitude $F_{f i}^{t}$ are dominated by four processes: i) $3 s \nu_{i} d \rightarrow 3 s \in f$ (a B-C transition with transition amplitude given by $F_{f i} \sim<\xi_{\nu_{\mathrm{i}} d}|r| \xi_{\epsilon f}>$ ), ii) $3 s \nu_{i} d \rightarrow 3 p \nu d$ (a shakeup B-B

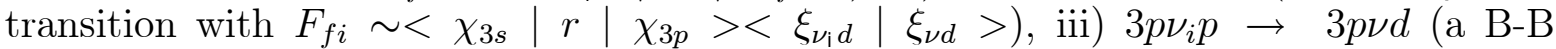
transition with $F_{f i} \sim<\xi_{\nu_{\mathrm{i}} p}|r| \xi_{\nu d}>$ ), and iv) $3 p \nu_{i} f \rightarrow 3 p \nu g$ (a B-B transition with $\left.F_{f i} \sim<\xi_{\nu_{i} f}|r| \xi_{\nu g}>\right)$. The outgoing photoelectron is represented by the effective oneparticle radial function $\xi_{\epsilon f}$. The outer $\nu \ell$ electron of the bound component of the $3 p \nu \ell^{1} F$ resonance is similarly represented by a radial function $\xi_{\nu \ell}$. For transition from the highly excited $3 s \nu_{i} d^{1} D$ states, the spectra are dominated by the $\Delta \nu=0$ shakeup process of the outer d electron following the $3 s \rightarrow 3 p$ core electron excitation. According to our BSCI calculation (Fang et al, 1997), a detailed breakdown of individual contributions to total $F_{f i}^{t}$, shown in Fig. 4 for the four processes listed earlier, indicates a strong dominance of the B-B shakeup process in the $A l^{+} 3 s 6 d^{1} D \rightarrow 3 p n l^{1} F$ photoionization. Specifically, its transition amplitude is given by $<\chi_{3 s}|r| \chi_{3 p}><\xi_{\nu_{\mathrm{i}} d} \mid \xi_{\nu d}>$. Whereas the effective orbital function $\xi_{6_{i} d}$, which represents the outer $6 d$ electron of the initial state, is energy independent, the effective orbital function $\xi_{6 d}$ for the outer $6 \mathrm{~d}$ electron of the broad $3 p 6 d^{1} F$ resonance varies significantly as energy varies near the narrow $3 p n g{ }^{1} F$ resonance. In fact, as shown in Fig. 5, similar to the sign change of the oscillating function $\xi_{\epsilon f}$ for the outgoing photoelectron (due to the increase of the scattering phase shift by a total of $\pi$ across the resonance), the sign of $\xi_{6 d}$ is also reversed as energy increases across the narrow $3 p n g{ }^{1} F$ resonance at $E_{r}=0.379975 R y$. This sign change in $\xi_{6 d}$ leads directly to a sign change in the overlapping integral $<\xi_{6 \mathrm{i} d} \mid \xi_{6 d}>$, i.e., a zero in the total transition amplitude $F_{f i}^{t}$, and ultimately the narrow $3 p n g{ }^{1} F$ window resonance. Also shown in Fig. 5, as expected, the energy-dependent amplitudes of 
both $\xi_{6 d}$ and $\xi_{\epsilon f}$ reach a near-zero minimum as the $3 p g$ configuration series accounts essentially $100 \%$ of the spectral density at the $3 p n g^{1} F$ resonance near $E_{r}$.

\subsection{Variation of structure profiles along the autoionization series}

For transition from bound excited states, our BSCI calculation has shown that the photoionization structure profiles could vary substantially along the autoionization series. For example, Fig. 6 shows that in photoionization from excited $3 s \nu_{i} d^{1} D$ states of Mg-like $\mathrm{Al}^{+}$, the cross sections near the neighboring $3 p \nu^{\prime} g^{1} \mathrm{~F}$ resonances on the higher energy side of the dominating $\Delta \nu=03 s \nu_{i} d^{1} D \rightarrow 3 p \nu d^{1} F$ resonance are consistently greater than the ones on the lower energy side. Similar feature is also found in spectra such as the ones in the $3 s \nu_{i} p{ }^{1} P \rightarrow{ }^{1} D$ photoionization of $\mathrm{Mg}$ (e.g., see Fig. 9 of Fang et al, 1997). A breakdown of the individual contributions to the total transition amplitude $F_{f i}^{t}$ from the four processes discussed earlier for the $A l^{+} 3 s 6 d^{1} D \rightarrow{ }^{1} F$ photoionization near the $3 p 5 l$ and $3 p 7 l{ }^{1} F$ resonances is shown in detail in Fig. 7. It shows that the transition amplitude for the $3 s \nu_{i} d \rightarrow 3 s \epsilon f$ B-C direct transition, i.e., $\left\langle\xi_{\nu_{i} d}|r| \xi_{\epsilon f}>\right.$, changes its sign twice due to the sign change of the effective oscillating function $\xi_{\epsilon f}$ as the scattering phase shift increases by a total of $\pi$ twice as energy increases across the $3 p \nu d$ and $3 p \nu g{ }^{1} F$ resonances, respectively.

For the $3 s \nu_{i} d \rightarrow 3 p \nu d \mathrm{~B}-\mathrm{B}$ shakeup process, the energy variation of the transition amplitude $F_{f i}$ is determined by the overlapping integral $O_{\nu \nu_{\mathrm{i}}}=<\xi_{\nu_{\mathrm{i}} d} \mid \xi_{\nu d}>$ between the effective radial functions of the initial and final $d$ electrons. Following our earlier discussion, the integral $O_{\nu \nu_{\mathrm{i}}}$ changes its sign due to the sign change of the effective final state radial function $\xi_{\nu d}$ at the $3 p \nu g{ }^{1} F$ resonance. In addition, the final state radial functions $\xi_{\nu d}$ expands slowly outwards as energy increases. Except for the $\Delta \nu=0$ transition, $O_{\nu \nu_{\mathrm{i}}}$ changes its sign at an energy close to the center of the $3 p \nu d{ }^{1} F$ resonance due to the quasi-orthogonality between the initial and effective final state radial functions of the outer $d$ electrons as shown in Fig. 8. In other words, whereas the transition amplitude $F_{f i}$ for the B-B shakeup process changes sign only once near the $3 p \nu g{ }^{1} F$ resonance for a $\Delta \nu=0$ transition, it changes sign twice for all $\Delta \nu \neq 0$ transitions, i.e., once at the $3 p \nu{ }^{1} F$ resonance due to the sign change of the radial function $\xi_{\nu d}$ of the outer $d$ electron and a second time near the $3 p \nu d{ }^{1} F$ resonance due to the quasi-orthogonality between the initial and final state effective radial functions. At energies near the broad $3 p 7 d{ }^{1} F$ resonance above the dominating shake-up structure, the constructive contribution due to the B-B $3 s \nu_{i} d \rightarrow 3 p \nu d$ shakeup process does not qualitatively change the general features of the total transition amplitude $F_{f i}^{t}$ from the $3 s \nu_{i} d \rightarrow 3 s \epsilon f$ direct B-C transition. Consequently, the $3 p 7 d{ }^{1} F$ resonance is characterized by a typical window resonance with a near-zero $q$ parameter. In contrast, in the vicinity of the $3 p 5 l^{1} \mathrm{~F}$ resonances, the transition amplitude is strongly affected by the destructive interference between the B-C and B-B contributions as shown in Fig. 7. As a result, the change in resonant features below and above the $\Delta \nu=0$ dominating shakeup structure can be attributed directly to the switch from destructive to constructive interference due to the difference in the number of sign changes between the transition amplitudes of the B-B and B-C processes as energy increases.

Another example which exhibits strong variation in resonant profile, based on our BSCI calculation, is found in photoionization from He $1 s 2 s{ }^{1} S$ metastable state leading to 
the doubly excited $s p, 2 n^{+}$autoionization series (Fang and Chang, 1997). The calculated $q$ parameter of the $s p, 2 n^{+}$series varies from a large positive value for the $2^{+}$state to a negative value of -8 for the $3^{+}$state. It returns to a positive value of 3.15 for the $5^{+}$ state after it reaches a near-zero value of 0.02 for the $4^{+}$state, resulting into a $s p, 24^{+}{ }^{1} \mathrm{P}$ window resonance shown in Fig. 9. The photoionization from the $1 s 2 s{ }^{1} S$ metastable state leading to the $s p, 22^{+}{ }^{1} P$ resonance is dominated by the one-electron $1 s \rightarrow 2 p$ transition. All other $1 s 2 s{ }^{1} S \rightarrow s p, 2 n^{+1} P$ transitions involve the orbital change of both electrons and the cross sections for transitions to the $3^{+}$or higher states are considerably smaller than the ones to the $2^{+}$state. The total transition amplitude $F_{f i}^{t}$ from an initial state $i$ to a final state $f$ is dominated by a direct $2 s \rightarrow \epsilon p$ B-C transition. From Eq. (3), the cross section $\sigma$ is proportional to $\left|F_{f i}^{t}\right|^{2}$ and inversely proportional to the square of the amplitude $A$ of the effective oscillating function of the outgoing ionized electron, i.e.,

$$
\sigma \sim \frac{\left|F_{f i}^{t}\right|^{2}}{A^{2}}
$$

Accordingly, the zero cross section can be linked directly to the zero in $F_{f i}^{t}$ and the peak of the resonance should be located at or near the minimum in $A^{2}$. The resonance structure is represented by a negative $q$-parameter when the zero in $F_{f i}^{t}$ is located on the higher energy side of the minimum in $A^{2}$. In contrast, if the zero in $F_{f i}^{t}$ is located on the lower energy side of the minimum in $A^{2}$, the $q$-parameter should have a positive value. In addition, the window resonance can be linked to a zero in $F_{f i}^{t}$ at an energy where $A^{2}$ approaches a minimum. Fig. 10 presents the total transition amplitudes $F_{f i}^{t}$ for the $1 s 2 s^{1} S \rightarrow s p, 2 n^{+}{ }^{1} P$ photoionization (the solid curve) and $A^{2}$ (the dotted curve) as functions of the photoelectron energy. Although both $F_{f i}^{t}$ and $A^{2}$ vary only modestly along the $s p, 2 n^{+1} P$ series, a small change in the relative position of the zeros in $F_{f i}^{t}$ and the minima in $A^{2}$ result in a strong variation in structure profile along the $s p, 2 n^{+}{ }^{1} P$ autoionization series shown in Fig. 9.

\subsection{Photoionization leading to multiple continua}

Non-relativistically, at an energy between the first and second ionization thresholds, the spectra of a light alkaline-earth atom leading to a single channel photoionization, are dominated by two strongly energy-dependent doubly excited asymmetric autoionization series, one broad and one narrow in width, due to the simultaneous change of electronic orbitals of two outer electrons. At an energy above the second ionization threshold, in contrast, the width of a doubly excited resonance is usually broader than the narrow resonances below the second ionization threshold, in part due to the presence of multiple ionization pathways. The resonance profiles for the overlapping doubly excited autoionization series are expected to be less regular due to the interference between transitions into multiple ionization channels.

In a recent BSK calculation (Fung et al, 2001), three doubly excited autoionization series, i.e., $4 s n p, 3 d n p$ and $3 d n f^{1} P$ between the $\mathrm{Mg}^{+} 3 p^{2} P$ and $\mathrm{Mg}^{+} 4 s^{2} S$ thresholds, 
shown schematically in Fig. 1 of Fung et al (2001), were studied in detail. The calculated BSK ground state photoionization cross sections of Mg from $78.5 \mathrm{~nm}$ to $88.5 \mathrm{~nm}$, shown in Fig. 11, are in good agreement with the measured photoabsorption cross sections. One of the more interesting findings of the BSK calculation is a hidden $3 p 4 d{ }^{1} P$ resonance near $83.5 \mathrm{~nm}$. Its presence, visually missing both in theoretical and experimental total cross section spectra, is present clearly in the theoretical partial cross section spectra at least in two of the ionization channels (i.e., $3 p \epsilon s$ and $3 p \epsilon d^{1} P$ ) shown in Fig. 5 of Fung et al (2001). In addition, the BSK calculation shows clearly that the $3 p 4 d^{1} P$ resonance can be identified unambiguously if the photoionization is originated from the bound excited $3 s 4 s{ }^{1} S$ state (Fig. 6 of Fung et al, 2001).

As expected, the resonance structure profiles above the second ionization threshold, even for ground state photoionization, vary substantially along the same autoionization series (see, e.g., Fig. 7 of Fung et al, 2001). When the resonances are well separated, a simple estimate of the effective principal quantum numbers against the $4 s$ and $3 d$ thresholds, based on the observed spectra, is sufficient to identify the individual member of the resonance series. But, when two or more resonances overlap substantially in energy, a detailed theoretical analysis is needed for an unambiguous identification of the individual resonances. Above the Mg 3p threshold, from the estimated effective principal quantum numbers of the $4 s \nu p$ and $3 d \nu f$ series against the $4 s$ and $3 d$ thresholds, respectively, suggest that there should exist one member from each of these two autoionization series at an energy close to $77.6 \mathrm{~nm}$. However, both observed and theoretical spectra presented in Fig. 7 of Fung et al (2001) show the presence of only one asymmetric resonance structure visible near $77.6 \mathrm{~nm}$. A detailed analysis based on the BSK calculation confirms a nearly degenerate overlap between a broad $3 d 5 f$ and a narrow $4 s 8 p$ resonances near $77.6 \mathrm{~nm}$. In fact, Fig. 12 shows that a narrow $4 s 8 p$ resonance can be identified from the $3 p \epsilon s$ partial cross sections spectrum. This narrow resonance is located near the center of a broad $3 d 5 f$ resonance, leading to an appearance of a single resonance in the total cross section. The close proximity of these two resonances can also be seen from the energy variation of the sum of the eigenphase shifts over all eigenchannels, $\eta_{t o t}$. Fig. 13 shows, unlike the increase in $\eta_{t o t}$ a value of $\pi$ for all other resonances, an increase of a value close to $2 \pi$ in $\eta_{t o t}$ between $E=-0.496$ Ry and -0.489 Ry (i.e., at wavelengths centered around $77.6 \mathrm{~nm}$ ) due to the presence of the overlapping $3 d 5 f$ and $4 s 8 p$ resonances.

The BSK results discussed above have also been confirmed recently using the BSCR method (Fang et al, 2002). As we pointed out earlier, the success of the BSCR method depends critically on the stability of the decay factor $\beta$ as the complex argument $\theta$ varies. Fig. 14 shows the variations of $E_{r}$ and $\Gamma$ of the $\mathrm{Mg} 4 s 4 p^{1} P$ and $3 d 4 p^{1} P$ resonances at $\theta=0.4$ radians as $\beta$ changes from 0.7 to 0.84 . The resonance energy $E_{r}$ and the resonance width $\Gamma$, determined at $\beta=\beta_{O}$ according to Eq. (16), stay essentially the same as $\theta$ varies. The results from both the BSK and BSCR methods are generally consistent with other existing theoretical calculations. Detailed numerical data are listed in Table 1 of Fang et al (2002). 


\section{Concluding remarks}

We have demonstrated in this paper that the B-spline based methods, as we envisioned over a decade ago, have indeed offered an effective theoretical approach in the study of atomic photoionization dominated by two strongly interacting outer shell electrons. Its ability to interpret quantitatively the individual transition based on the explicit wave function information extracted from massive configuration interaction calculation has made it a very attractive alternative to other theoretical approaches.

For a more complicated divalent atom, such as $\mathrm{C}$ and $\mathrm{Si}$, the energy required for transitions from the ground state to low-lying excited states with a inner shell vacancy is relatively small in comparison with other type of inner shell process. For example, the excitation energy for $\mathrm{Si}$ atom from its $3 \mathrm{~s}^{2} 3 \mathrm{p}^{2}{ }^{3} P$ ground state to the highly-correlated $3 \mathrm{~s} 3 \mathrm{p}^{3}{ }^{3} \mathrm{D}$ excited state is approximately $6.0 \mathrm{eV}$. (The $3 \mathrm{~s} 3 \mathrm{p}^{3}{ }^{3} \mathrm{D}$ state is mixed strongly with the $3 \mathrm{~s}^{2} 3 \mathrm{p} 3 \mathrm{~d}^{3} D$ and $3 \mathrm{~s}^{2} 3 \mathrm{p} 4 \mathrm{~d}^{3} D$ states located at about $6.7 \mathrm{eV}$ and $7.1 \mathrm{eV}$ above the ground state, respectively.) As a result, an optimized CI wavefunction, with configurations representing three or more excited orbitals from two subshells, is needed to account for transitions involving three actively interacting electrons. The inclusion of such configurations also offers the opportunity for an explicit estimation of the contribution to the transition amplitude from long range core-polarization. Extension of the B-spline based approaches for such a transition is currently in progress. A recent BSCI application, which includes explicitly configurations corresponding to three excited orbitals, to the fine structure level splittings of four electron systems have yielded very good results that are substantially better than a similar BSCI calculation limited only to two excited orbitals.

The emphasis on the quantitative estimations of the multi-electron interactions requires well-planed numerical efforts. We estimate an increase of about one order of magnitude in computational effort for every additional excited orbital included explicitly in the theoretical calculation. With recent rapid advance in inexpensive PC cluster and a relatively simple numerical algorithm required in the B-spline based approaches, we anticipate a manageable computational effort as we move to a detailed investigation of atomic transitions involving the active participation of more than two electrons.

This work was supported by NSF under Grant No. PHY9802557 and the National Science Council of Taiwan under Grant No. NSC 91-2112-M030-002. 


\section{References}

Altun, Z., Manson, S.T., 1996. Photoionization of atomic scandium in the 3p to 3d resonance region. Europhys. Lett. 33, 17-22.

Aymar, M., Greene, C.H., Luc-Konnig, E., 1996. Multichannel Rydberg spectroscopy of complex atoms. Rev. Mod. Phys. 68, 1015-1123.

Bartschat, K., Hudson, E.T., Scott, M.P., Burke, P.G., Burke, V.M., 1996. Electronatom scattering at low and intermediate energies using a pseudo-state/R-matrix basis. J. Phys. B29, 115-123.

Bell, R.H., Seaton, M.J., 1985. Dielectronic recombination: I. General theory. J. Phys. B18, 1589-1629.

Brage, T., Froese Fischer, C., and Miecznik, G., 1992. Non-variational, spline-Galerkin calculations of resonance positions and widths, and photodetachment and photoionization cross sections for $\mathrm{H}^{-}$and He. J. Phys. B25, 5289-5314.

Burke, P.G., 1993. R-matrix Theory of Atomic and Molecular Process. In: Chang, T.N. (Ed.), Many-body Theory of Atomic Structure and Photoionization, World Scientific, Singapore, pp. 47-82.

Carravetta, V., Spizzo, P., Moccia, R., 1993. L ${ }^{2}$ Basis Function Methods for the Electronic Continuum. Photoionization and some Related Processes. In: Chang, T.N. (Ed.), Many-body Theory of Atomic Structure and Photoionization, World Scientific, Singapore, pp. 175-212.

Chang, T.N, 1987. Effect of the configuration interaction on the theoretical oscillator strengths of the magnesium atom. Phys. Rev. A36, 447-455.

Chang, T.N., 1989a. Energy Levels and the Oscillator Strengths of Be Atom Determined by a Configuration-Interaction Calculation with Finite Basis Set from B Splines. Phys. Rev. A39, 4946-4955.

Chang, T.N., 1989b. Combined Perturbation and Configuration-interaction Calculation for the Fine-Structure Level Splittings of the 1s4f States of Helium. Phys. Rev. A39, 6129-6133.

Chang, T.N., 1990. Radiative Lifetimes of the Bound Excited States of Magnesium and Beryllium. Phys. Rev. A41, 4922-4929.

Chang, T.N., Mu, Y., 1990. Energy levels and transition probabilities of bound excited states of Be-like NIV. J. Quant. Spectrosc. Radiat. Transfer 44, 413-420.

Chang, T.N., Wang, R. Q., 1991a. Effect of Positive-energy Orbitals on the Configurationinteraction Calculation of the $\mathrm{H}^{-}$Atom. Phys. Rev. A43, 1218-1222. 
Chang, T.N., Wang, R. Q., 1991b. Nonuniform Variation of the Radiative Lifetimes of the Bound Excited States along the Mg Isoelectronic Sequence. Phys. Rev. A44, 80-89.

Chang, T.N., Tang, X., 1991. Photoionization of two-electron Atoms using a Nonvariational Configuration-interaction Approach with Discretized Finite Basis. Phys. Rev. A44, 232-238.

Chang, T.N., Tang, X., 1992. Atomic Structure Effects in Multiphoton ionization of magnesium. Phys. Rev. A46, R2209-R2212.

Chang, T.N., 1993a. Widths of the Doubly Excited Resonances of Two-electron Atoms Below the $\mathrm{n}=2$ Threshold. Phys. Rev. A47, 705-708.

Chang, T.N., 1993b. B-spline Based Configuration Interaction Approach for Photoionization of Two-electron and Divalent Atoms. In: Chang, T.N. (Ed.), Many-body Theory of Atomic Structure and Photoionization, World Scientific, Singapore, pp. 213-247.

Chang, T.N., Zhen, M., 1993. Photoionization from 1 sns ${ }^{1,3} S^{e}$ States of Helium. Phys. Rev. A47, 4849-4855.

Chang, T.N., Zhu, L., 1993. Resonant Width and Energy Determined by Photoionization from Excited Divalent Atoms. Phys. Rev. A48, R1725-R1728.

Chang, T.N., Zhu, L., 1995. Photoionization of the Be isoelectronic sequence from the ground and the ${ }^{1} S$ bound excited states. Phys. Rev. A51, 374-380.

Chen, M.H., Cheng, K.T., Johnson, W.R., 1993. Relativistic configuration-interaction calculations of $n=2$ triplet states of heliumlike ions. Phys. Rev. A47, 3692-3703.

Chen, M.K., Hsue, C.S., 1992. Variational calculations for high- ${ }^{3} S$ states in He with B-spline basis functions. J. Phys. B25, 4059-4067.

Chen, M.K., 1993. 1sns Rydberg states of the He atom. J. Phys. B26, 3025-3034.

Chung, K.T., Gou, B.C., 1995. Energies and lifetimes of triply excited states of lithium. Phys. Rev. A52, 3669-3676.

Cormier, E., Bachau, H., Zhang, J., 1993. Discretization techniques applied to the study of multiphoton excitation of resonances in helium. J. Phys. B26, 4449-4463.

Decleva, P., Lisini, A., Venuti, M., 1994. Multichannel continuum states by a leastsquares approach in a spline basis: application to $\mathrm{He}$ and $\mathrm{H}^{-}$photoionization. J. Phys. B27, 4867-4889.

de Boor, C., 1978. A practical guide to splines, Springer-Verlag, New York.

Du, N.Y., Starace, A.F., Cherepkov, N.A., 1993. Resonant two-color detachment of $\mathrm{H}^{-}$ with excitation of $\mathrm{H}(\mathrm{n}=2)$. Phys. Rev. A48, 2413-2425. 
Dubau, J., Wells, J., 1973. Quantum defect theory X. Photoionization. J. Phys. B6, 1452-1460.

Fang, T.K., Nam, B.I., Kim, Y.S., Chang, T.N., 1997. Resonance Structures of Overlapping Doubly Excited Autoionization Series in Photoionization of $\mathrm{Mg}^{-l i k e ~} \mathrm{Al}^{+}$and $\mathrm{Si}^{+2}$ Ions. Phys. Rev. A55, 433-439.

Fang, T.K., Chang, T.N., 1997. Doubly Excited Resonance Structure in He Photoionization from $1 s 2 s^{1,3} S$ Metastable States. Phys. Rev. A56, 1650-1653.

Fang, T.K., Ho, Y.K., 1999. Strong electric-field effects on the structure profiles of doubly excited resonances in He ground-state photoionization. Phys. Rev. A60, 21452150 .

Fang, T.K., Chang, T.N., 2000. B-spline Based Multichannel K-matrix Method for Atomic Photoionization. Phys. Rev. A61, 062704-(1-7).

Fang, T.K., Ho, Y.K., Lin, Y.C., 2002. B-splined-based complex-rotation method for two-electron and divalent atoms, In: Proceedings of International Seminar on Photoionization in Atom, Koyto, Japan, pp. 12-17.

Fano, U., 1961. Effects of Configuration Interaction on Intensities and Phase Shifts, Phys. Rev. 124, 1866-1878.

Froese Fischer, C., Saha, H.P., 1987. Photoionization of magnesium. Can J. Phys. 65, $772-776$.

Froese Fischer, C., Idrees, M., 1990. Spline methods for resonances in photoionisation cross sections. J. Phys. B23, 679-691.

Froese Fischer, C., 1993. Non-variational Multiconfiguration Hartree-Fock Claculations for Continuum Wave Functions. In: Chang, T.N. (Ed.), Many-body Theory of Atomic Structure and Photoionization, World Scientific, Singapore, pp. 143-173.

Fung, H.S., Wu, H.H., Yih, T.S., Fung, T.K., Chang, T.N., 2001. Photabsorption of Mg above the 3p threshold. Phys. Rev. A64, 052716-(1-5).

Greene, C.H., Kim, L, 1987. Two-electron excitations in atomic calcium. Phys. Rev. A36, 2706-2717.

Ho, Y.K., 1983. The method of complex coordinate rotation and its application to atomic collision processes. Phys. Rep. 99, 1-68.

Hudson, E.T., Bartschat, K., Scott, M.P., Burke, P.G., Burke, V.M., 1996. Electron scattering from helium atoms: phase shifts, resonance parameters and total cross sections. J. Phys. B29, 5513-5526.

Johnson, W.R., Blundell, S.A., Sapirstein, J., 1988. Finite basis sets for the Dirac equation constructed from B splines. Phys. Rev. A37, 307-315. 
Kim, D.S., Kim, Y.S., Zhou, H.L., Manson, S.T., 1997a. Photoelectron angular distribution of the $2 \mathrm{p}$ subshell for the $1 \mathrm{~s} 2 \mathrm{~s} 2 \mathrm{p}{ }^{4} \mathrm{P}$ state of $\mathrm{He}^{-}, \mathrm{Li}$ and $\mathrm{Be}^{+}$. J. Phys. B30, 3379-3386.

Kim, D.S., Zhou, H.L., Manson, S.T., 1997b. Photodetachment of the 1s2s2p ${ }^{4} \mathrm{P}$ state of $\mathrm{He}^{-}$from threshold to $100 \mathrm{eV}$. Phys. Rev. A55, 414-425.

Lee, C.M., Lu, K.T., 1973. Spectroscopy and collision theory. II. The Ar absorption spectrum. Phys. Rev. A8, 1241-1257.

Lin, C.D., 1976. Properties of resonance state in $\mathrm{H}^{-}$. Phys. Rev. A14, 30-35.

Lin, C.D., 1984. Classification and supermultiplet structure of doubly excited states. Phys. Rev. A29, 1019-1033;

Lin, C.D., 1986. Doubly excited states, including new classification schemes. Adv. At. Mol. Phys. 22, 77-142.

Liu, C.R., Gao, B., Starace, A.F., 1992. Variationally stable treatment of two- and three-photon detachment of $\mathrm{H}^{-}$including electron-correlation effects. Phys. Rev. A46, 5985-5998.

Lu, K.T., Fano, U., 1970. Graphic analysis of perturbed Rydberg series. Phys. Rev. A2, 81-86.

Lu, K.T., 1971. Spectroscopy and collision theory. The Xe absorption spectrum. Phys. Rev. A4, 579-596.

Macek, J., 1968. Properties of autoionizing states of He. J. Phys. B1, 831-843.

Mendoza, C., 1981. Term structure of Mg I calculated in a frozen-cores approximation. J. Phys. B14, 397-409.

Miecznik, G., Brage, T., Froese Fischer, C., 1993. Autoionization studies of the 1s2s2p ${ }^{4} P_{5 / 2}$ levels in $\mathrm{He}^{-}, \mathrm{Li}$ I, and Be II. Phys. Rev. A47, 3718-3723.

Moccia, R., Spizzo, P., 1985. One-photon transition probabilities and photoionisation cross section calculations of Be. J. Phys. B18, 3537-3554.

Moccia, R., Spizzo, P., 1987. An $\mathrm{L}^{2}$ calculation of the ${ }^{1,3} P^{o}$ resonances of atomic helium. J. Phys. B20, 1423-1431.

Moccia, R., Spizzo, P., 1990. Lithium anion photodetachment up to the 3s threshold: a K-matrix $\mathrm{L}^{2}$ basis calculation. J. Phys. B23, 3557-3567.

Moccia, R., Spizzo, P., 1991. Helium photoionization between $\mathrm{N}=2$ and $\mathrm{N}=3$ threshold including angular distribution and resonance preperties: A K-matrix $L^{2}$ basis-set calculation. Phys. Rev. A43, 2199-2214.

Norcross, D.W., Seaton, M.J., 1976. Energy levels for Be I calculated using a model potential and cores approximations. J. Phys. B9, 2983-3000. 
Pan, C., Starace, A.F., Greene, C.H., 1996. Photodetachment of $\mathrm{Li}^{-}$from the Li 3s threshold to the Li 6s threshold. Phys. Rev. A53, 840-852.

Rescigno, T.N., McCurdy, C.W. Jr., Orel, A.E., 1978. Extensions of the complexcoordinate method to the study of resonances in many-electron systems. Phys. Rev. A17, 1931-1938.

Rescigno, T.N., 1985. Atomic photoionization by the complex-basis-function expansion method: Application to ground-state and metastable Mg. Phys. Rev. A31, 607-611.

Rescigno, T.N., McCurdy, 1985. Locally complex distortions of the energy spectrum in the calculation of scattering amplitudes and photoionization cross sections. Phys. Rev. A31, 624-633.

Robicheaux, F., Greene, C.H., 1993a. Valence-shell photoabsorption spectra of C, Si, Ge, and Sn. Phys. Rev. A47, 4908-4919.

Robicheaux, F., Greene, C.H., 1993b. Photoionization of the scandium atom. I. General features, Phys. Rev. A48, 4429-4440.

Robicheaux, F., Gorczyca, T.W., Pindzolz, M.S., Badnell, N.R., 1995. Inclusion of radiation damping in the close-coupling equations for electron-atom scattering. Phys. Rev. A52, 1319-1333.

Sadeghpour, H.R., Greene, C.H., 1990. Dominant photodetachment channels in $\mathrm{H}^{-}$. Phys. Rev. Lett. 65, 313-316.

Seaton, M.J., 1966. Quantum defect theory. I. General formulation. Proc. Phys. Soc. $88,801-814$.

Starace, A.F., 1982. Theory of Atomic Photoionization. In: Mehlhorn, W. (Ed.), Handbuch der Physik, Springer-Verlag, Berlin, pp. 1-121.

Tang, J.Z., Watanabe, S., Matsuzawa, M., 1992. General computational method for two-electron systems. A46, 2437-2444.

Tang, J.Z., Watanabe, S., Matsuzawa, M., 1993. Very narrow doubly excited $2(1,0)^{-} \mathrm{n}$ and $2(-1,0)^{0} \mathrm{n}{ }^{1} P^{o}$ states of helium. Phys. Rev. A48, 841-844.

Tang, J.Z., Shimamura, I., 1995. Mechanism of the enhancement of some high-lying resonance series in the photoionization spectra of excited helium. Phys. Rev. A51, R1738-R1741.

Tang, X., Chang, T.N., Lambropoulos, P., Fournier, S., DiMauro, L.F., 1990. Multiphoton Ionization of Magnesium with Configuration Interaction Calculation. Phys. Rev. A41, 5265-5268.

Tang, X., Bachau, H., 1993. Three-photon ionization cross section calculations of Be. J. Phys. B26, 75-83. 
Thompson, D.J., Hibbert, A., Chandra, N., 1974. Photoionization of the (3s, 3p: $\left.{ }^{1} P\right)$ state of magnesium. J. Phys. B7, 1298-1305.

Vaeck, N., Godefroid, M., Hansen, J.E., 1991. MCHF oscillator strength and lifetime calculations in neutral calcium. J. Phys. B24, 361-381.

van der Hart, H.W., Hansen, J.E., 1992a. Calculations of double-excited states in He, $\mathrm{N}^{5+}$ and $\mathrm{N}^{3+}$ using B-splines. J. Phys. B25, 41-59.

van der Hart, H.W., Hansen, J.E., 1992b. Energies and autoionization widths of doublyexcited $3 \ln l^{\prime}$ states in $\mathrm{N}^{+5}$. J. Phys. B25, 2267-2278.

van der Hart, H.W., Hansen, J.E., 1993. Competition between radiative and nonradiative decay for doubly excited $2 \ln l^{\prime}$ and $3 \ln l^{\prime}$ states in $\mathrm{C}^{+4}$. J. Phys. B26, 641-662.

Wood, R.P., Greene, C.H., 1994. Asymmetric two-electron excitations in atomic strontium and barium, Phys. Rev. A49, 1029-1040.

Xi, J., Wu, L., Li, B., 1993. Correlation energies of Be-like atoms: A multimodel space many-body perturbation calculation with finite basis sets. Phys. Rev. A47, 2701-2709.

Zhou, B., Lin, C.D., 1994. Photoionization from metastable $(1 \mathrm{~s} 2 \mathrm{~s}){ }^{1} S^{e}$ and ${ }^{3} S^{e}$ states of the He atome for energies between the $\mathrm{N}=2$ and 3 thresholds of $\mathrm{He}^{+}$. Phys. Rev. A49, 1057-1065. 


\section{Figure captions}

Fig. 1. The energy variation of the effective oscillating orbital functions $\xi_{\epsilon p}(r)$ across the $2 p 5 s^{1} P$ resonance of Be-like $C$ III at $\epsilon=k^{2}=0.17989$ Ry. The dipole matrix element $d_{s p}=<\xi_{\epsilon p}|r| \xi_{2 s}>$ and the scattering phase shifts $\delta_{p}$ are also given (Chang and Zhu, 1995).

Fig. 2. The energy variation of the dipole matrix elements $\left\langle\xi_{\epsilon p}|r| \xi_{2 s}>\right.$ near the $2 p 5 s^{1} P$ resonance of Be-like $C$ III shown in Fig. 1.

Fig. 3. The $3 s 6 d^{1} D \rightarrow 3 p 6 d^{1} F$ photoionization of $A l^{+}$(Fang et al, 1997).

Fig. 4. The $A l^{+} 3 s 6 d^{1} D \rightarrow{ }^{1} F$ photoionization near the $3 p 6 d$ and $3 p 6 g$ resonances and the individual contributions $F_{f i}$ to the total transition amplitude $F_{f i}^{t}$ from processes i)-iv). Also given is the probability density $\rho_{3 s f}$, defined by Eq. (13) of Chang and Tang (1991), for the $3 s f$ ionization channel (Fang et al, 1997).

Fig. 5. The energy variation of $\xi 6 d$ and $\xi_{\epsilon f}$, defined by Eq. (10) of Chang and Tang (1991), near the $A l^{+} 3 p 6 d$ and $3 p 6 g^{1} F$ resonances (Fang et al, 1997).

Fig. 6. Photoionization cross sections $\sigma$ leading to the ${ }^{1} F$ continuum from the bound excited $3 s 6 d^{1} D$ state of Mg-like $\mathrm{Al}^{+}$(Fang et al, 1997).

Fig. 7. The $A l^{+} 3 s 6 d^{1} D \rightarrow{ }^{1} F$ photoionization near the $3 p 5 l$ and $3 p 7 l^{1} F$ resonances and the individual contributions $F_{f i}$ to the total transition amplitude $F_{f i}^{t}$ from processes i)-iv) (Fang et al, 1997).

Fig. 8. The energy variation of $\xi 5 d$ and $\xi_{7 d}$ near the $3 p 5 d^{1} F$ and $3 p 7 d^{1} F$ resonances, respectively, compares with the energy-independt effective initial radial function $\xi_{6 d}$. The overlapping integral $O_{\nu \nu_{\mathrm{i}}}=\left\langle\xi_{\nu_{\mathrm{i}} d} \mid \xi_{\nu d}\right\rangle$ changes sign near the resonance due to the quasi-orthogonality between the initial and effective final state radial functions of the outer $d$ electrons (Fang et al, 1997).

Fig. 9. Variation of the He $1 s 2 s{ }^{1} S \rightarrow{ }^{1} P$ photoionization resonance structures along the $s p, 2 n^{+}$autoionization series (Fang and Chang, 1997).

Fig. 10. The energy variation of the total transition amplitudes $F_{f i}^{t}$ (solid curve) and the square of the amplitude $A$ of the oscillating effective oneparticle functions representing the outgoing photoelectron from the $1 s 2 s^{1} S \rightarrow s p, 2 n^{+1} P$ photoionization (Fang and Chang, 1997).

Fig. 11. Comparison between measured absolute Mg photoabsorption cross sections and the theoretical photoionization cross sections using BSK approach from $78.5 \mathrm{~nm}$ to $88.5 \mathrm{~nm}$ (Fung et al, 2001).

Fig. 12. The overlapping narrow $\operatorname{Mg} 4 s 8 p^{1} P$ resonance and the broad $3 d 5 f^{1} P$ resonance near $77.6 \mathrm{~nm}$ (Fung et al, 2001).

Fig. 13. The energy variation of $\eta_{\text {tot }}$ where $E$ in $R y$ is measured against the $M g^{+2}$ threshold (Fung et al, 2001).

Fig. 14. The variations of $E_{r}$ and $\Gamma$ of the $\operatorname{Mg} 4 s 4 p^{1} P$ and $3 d 4 p^{1} P$ resonances for different $\beta$ values at $\theta=0.4$ radians (Fang et al, 2002). 


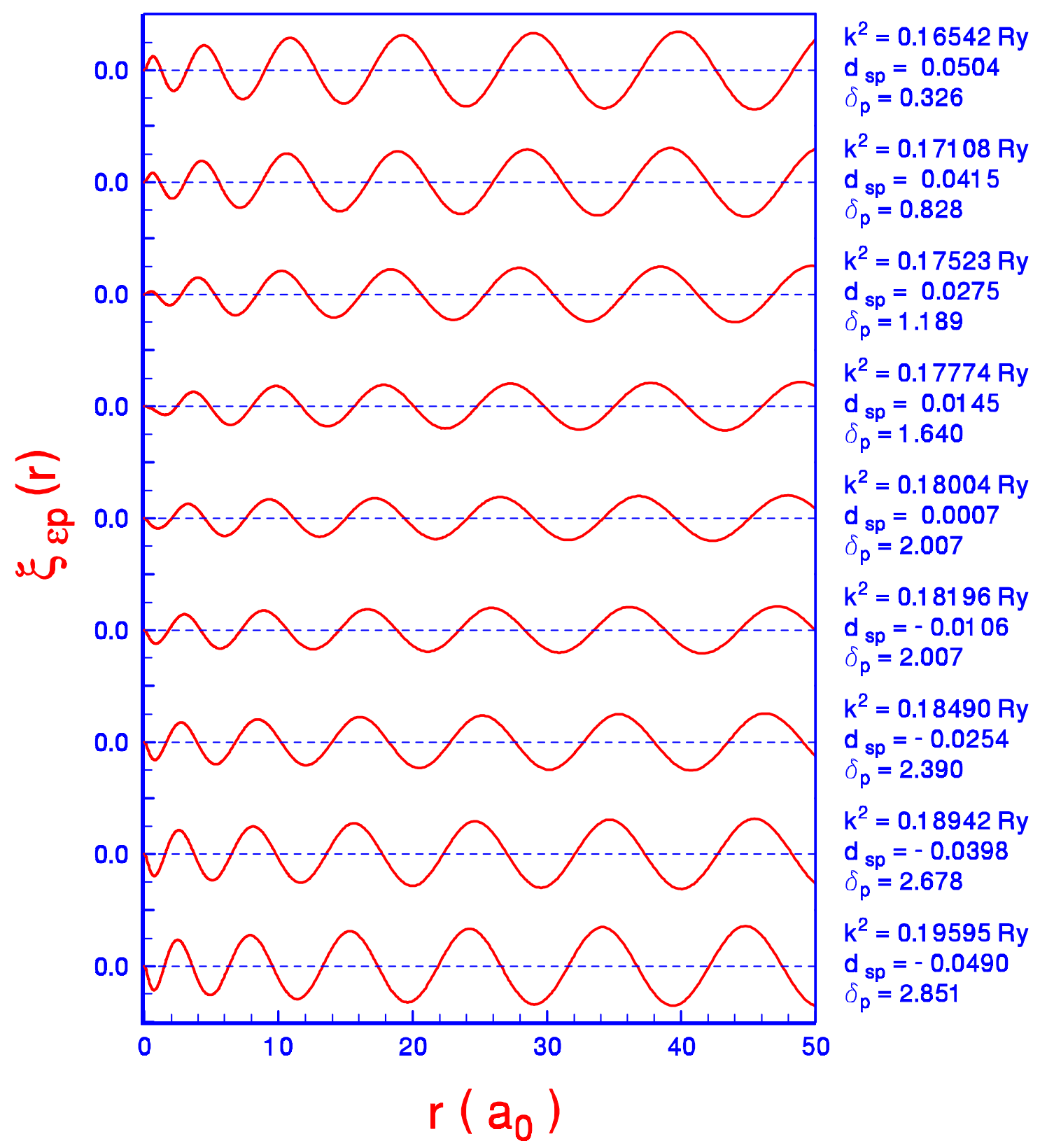

Fig. 1. The energy variation of the effective oscillating orbital functions $\xi_{\epsilon p}(r)$ across the $2 p 5 s^{1} P$ resonance of Be-like $C$ III at $\epsilon=k^{2}=0.17989$ Ry. The dipole matrix element $d_{s p}=<\xi_{\epsilon p}|r| \xi_{2 s}>$ and the scattering phase shifts $\delta_{p}$ are also given (Chang and Zhu, 1995). 


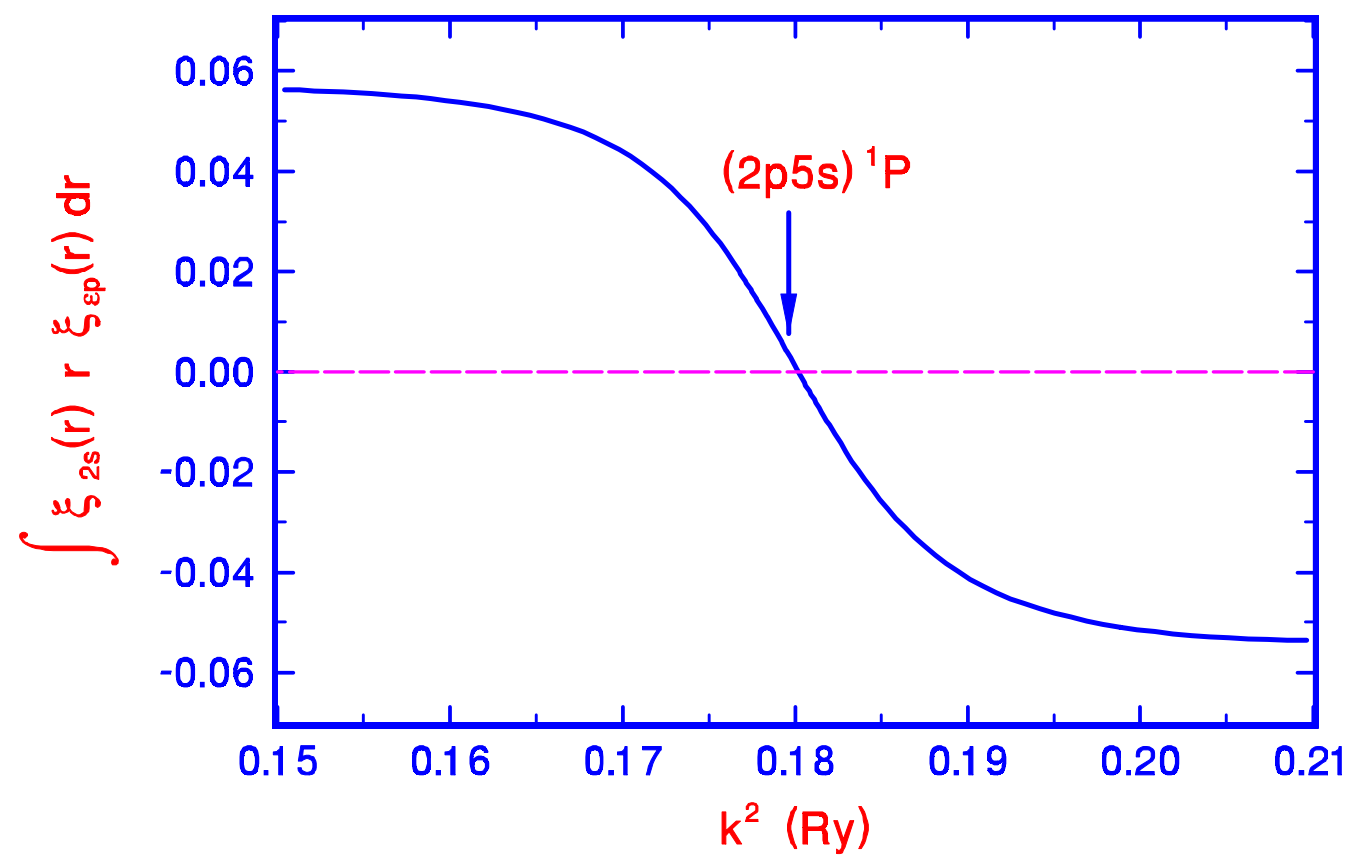

Fig. 2. The energy variation of the dipole matrix elements $<\xi_{\epsilon p}|r| \xi_{2 s}>$ near the $2 p 5 s^{1} P$ resonance of Be-like $C$ III shown in Fig. 1.

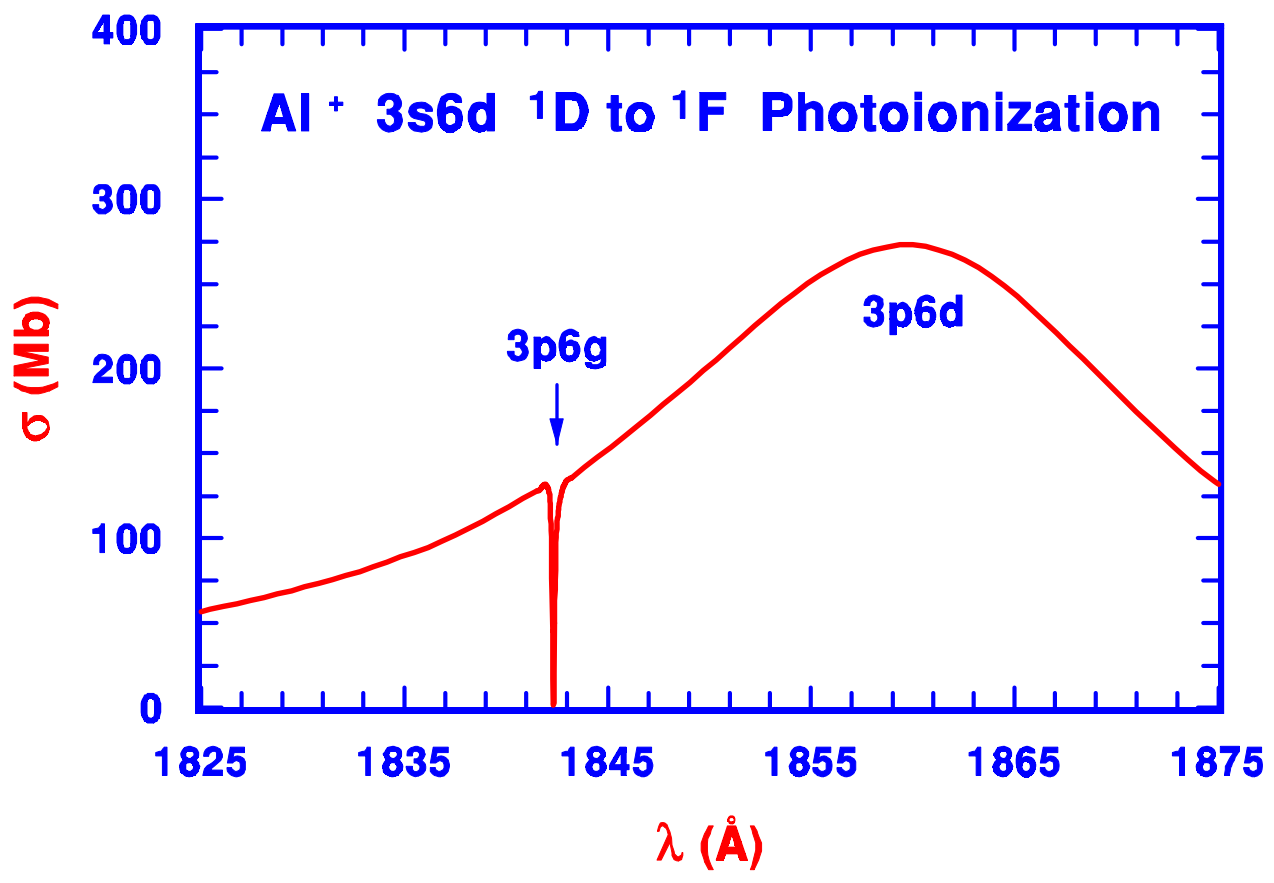

Fig. 3. The $3 s 6 d^{1} D \rightarrow 3 p 6 d^{1} F$ photoionization of $\mathrm{Al}^{+}$(Fang et al, 1997). 

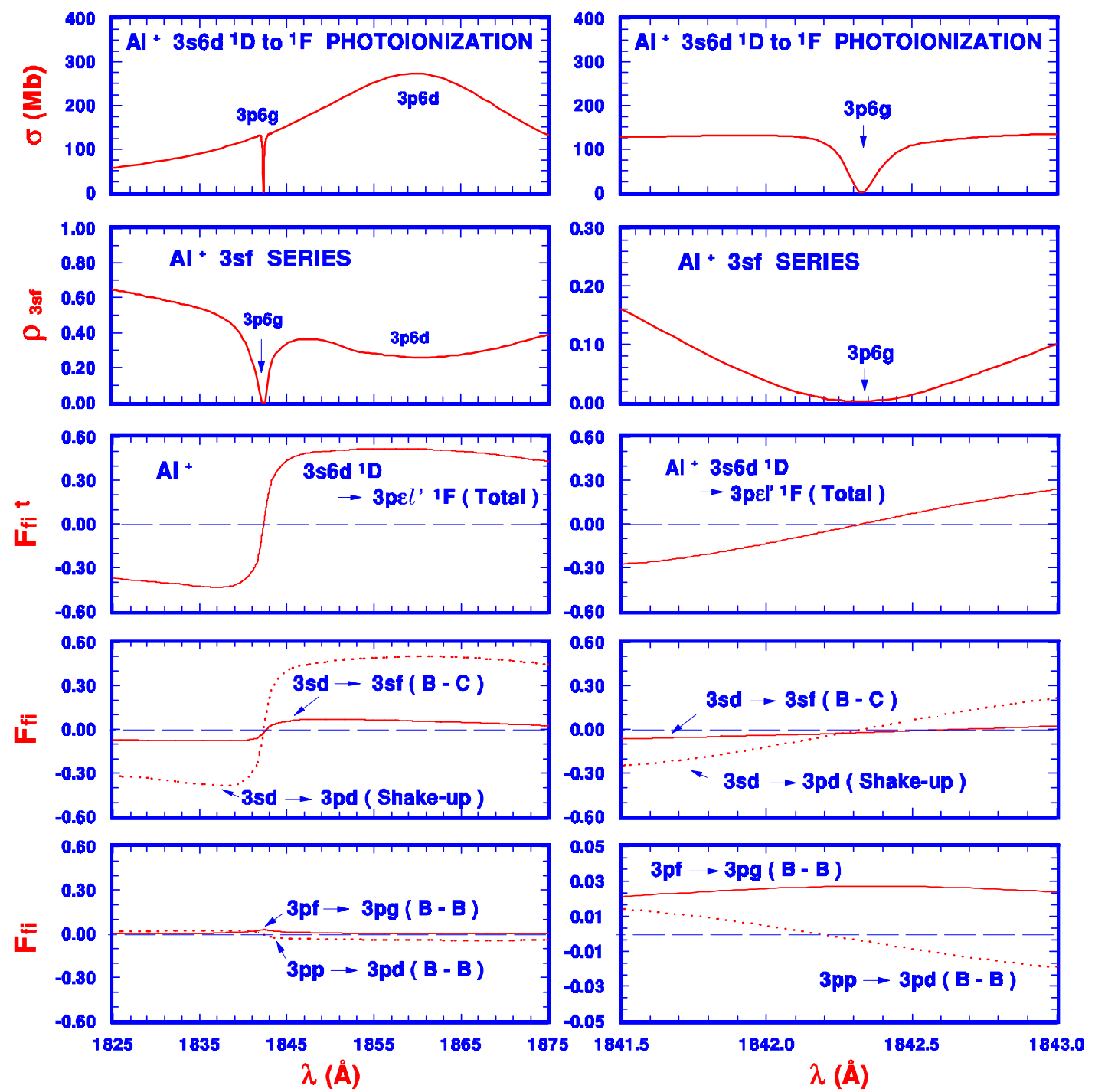

Fig. 4. The $A l^{+} 3 s 6 d^{1} D \rightarrow{ }^{1} F$ photoionization near the $3 p 6 d$ and $3 p 6 g$ resonances and the individual contributions $F_{f i}$ to the total transition amplitude $F_{f i}^{t}$ from processes i)-iv). Also given is the probability density $\rho_{3 s f}$, defined by Eq. (13) of Chang and Tang (1991), for the $3 s f$ ionization channel (Fang et al, 1997). 

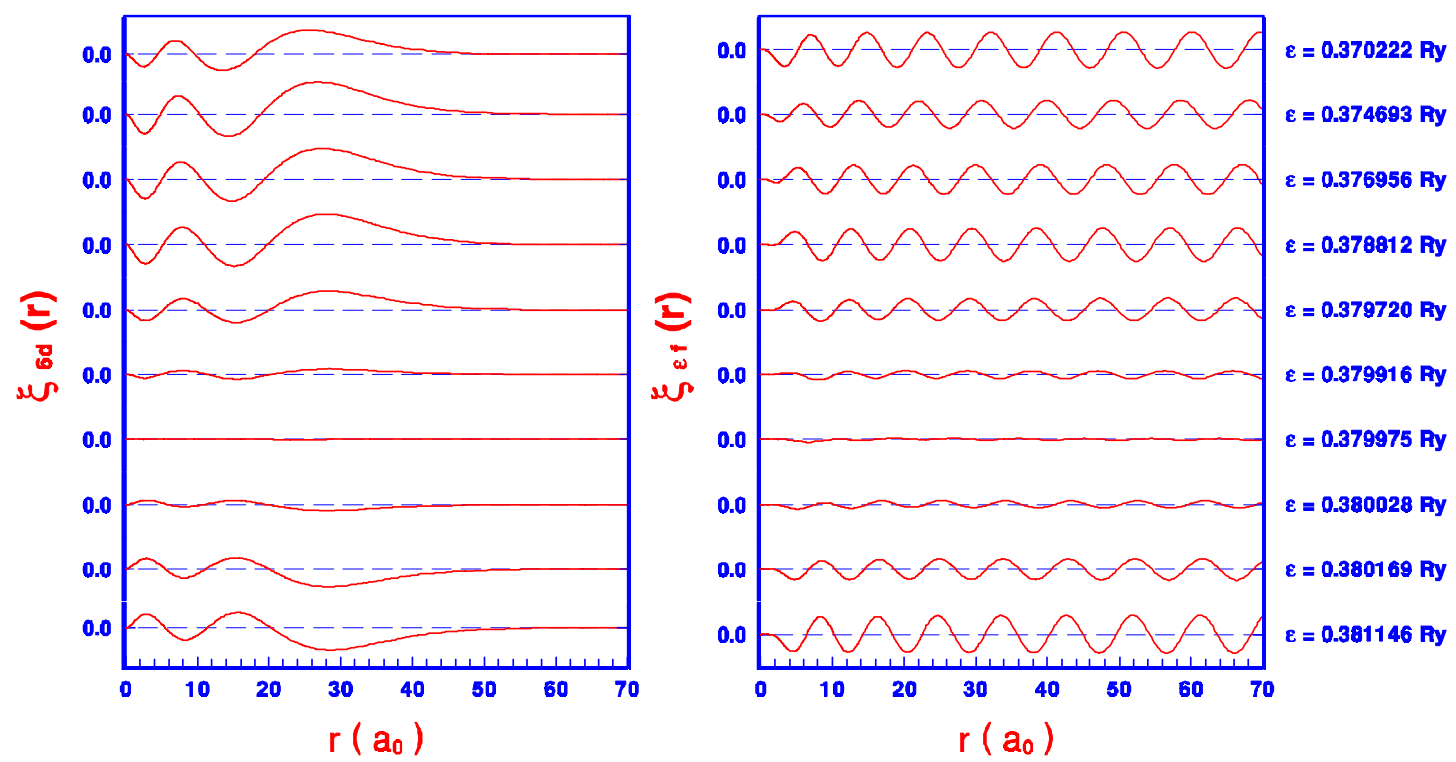

$$
\begin{aligned}
& E_{r}\left(3 p 6 d^{1} F\right)=0.375065 \pm 7.56 \times 10^{-3} R y \\
& E_{r}\left(3 p 6 g^{1} F\right)=0.379975 \pm 3.49 \times 10^{-5} R y
\end{aligned}
$$

Fig. 5. The energy variation of $\xi_{6 d}$ and $\xi_{\epsilon f}$, defined by Eq. (10) of Chang and Tang (1991), near the $A l^{+} 3 p 6 d$ and $3 p 6 g^{1} F$ resonances (Fang et al, 1997).

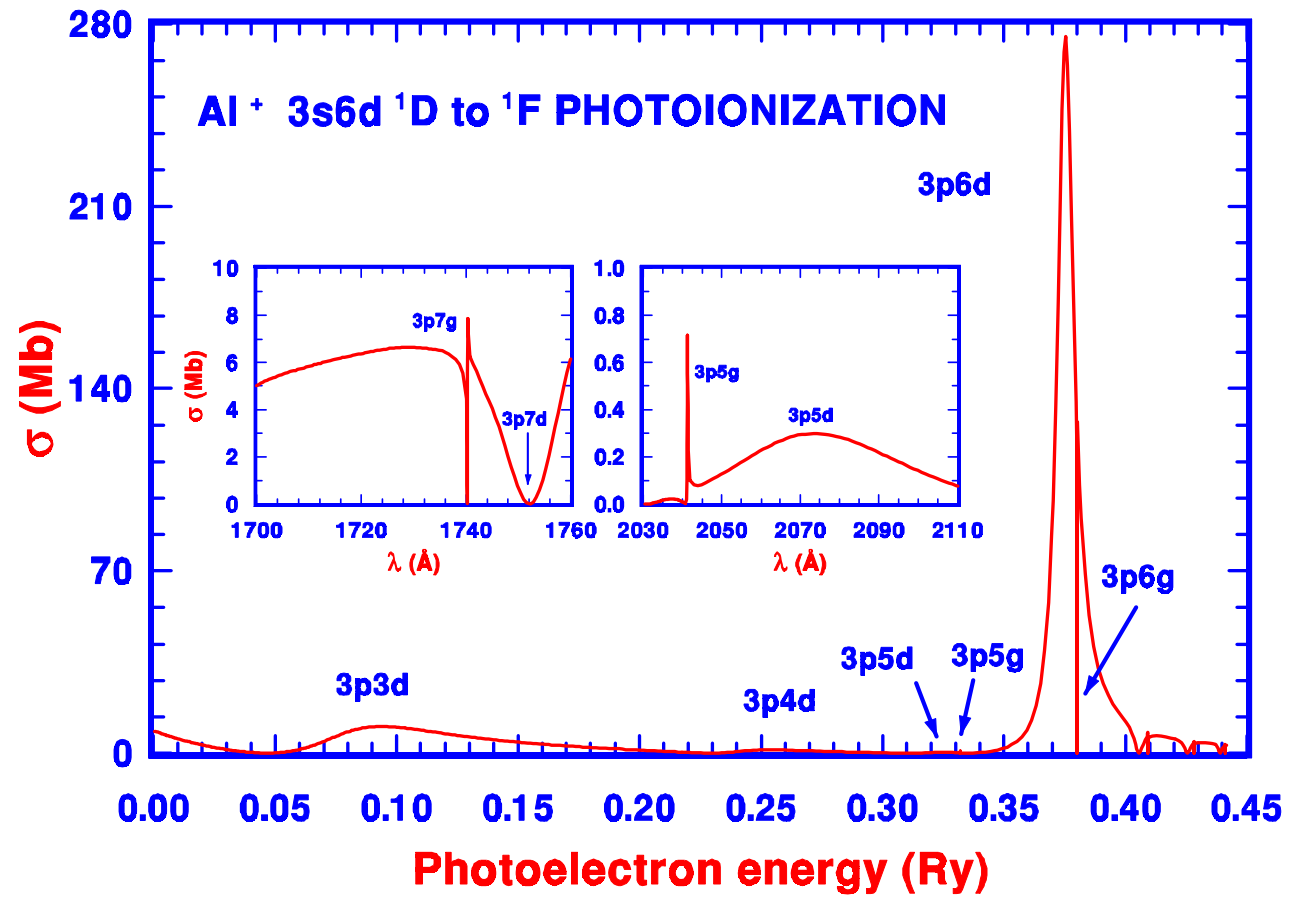

Fig. 6. Photoionization cross sections $\sigma$ leading to the ${ }^{1} F$ continuum from the bound excited $3 s 6 d^{1} D$ state of Mg-like $A l^{+}$(Fang et al, 1997). 

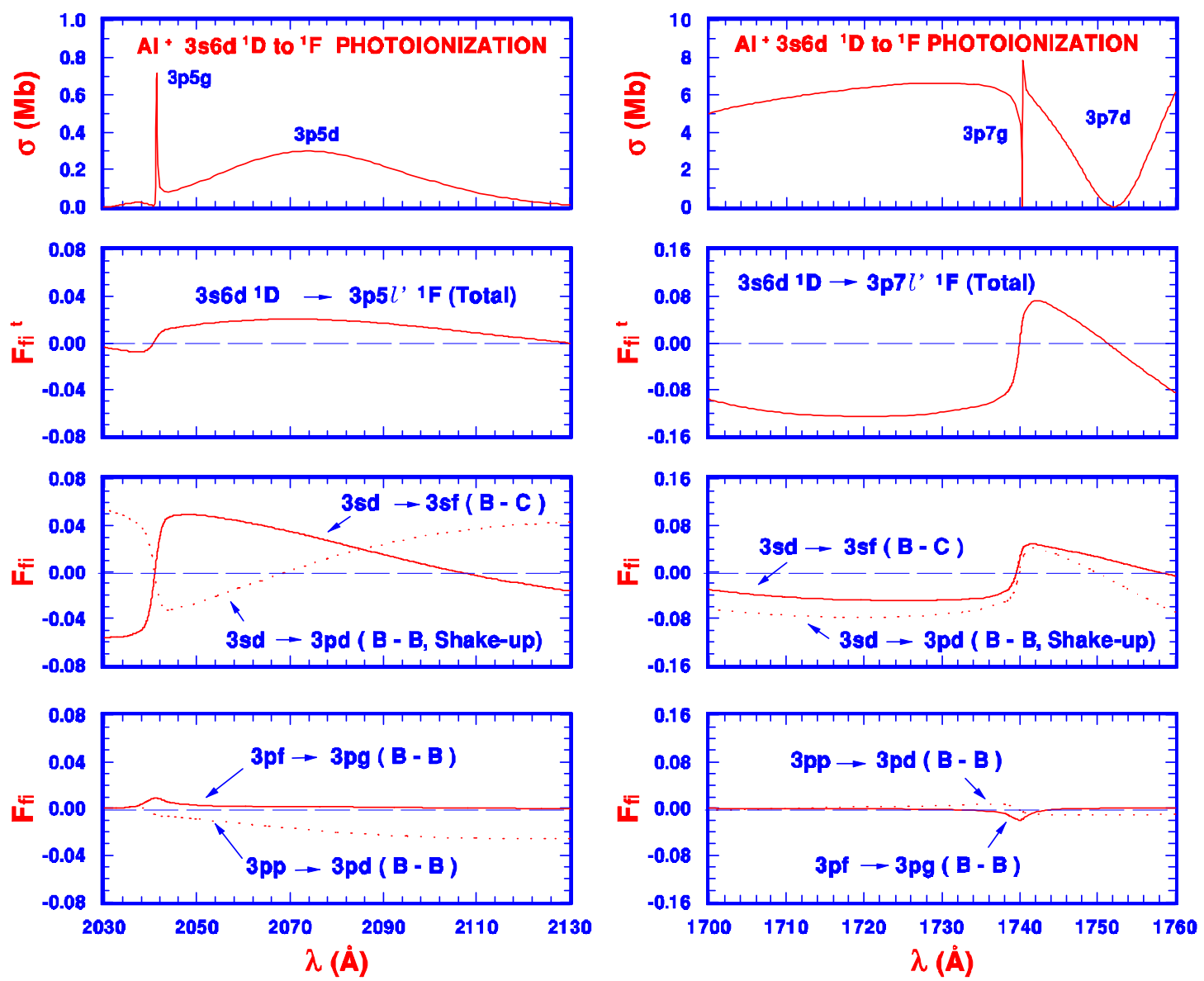

Fig. 7. The energy variation of $\xi_{5 d}$ and $\xi_{7 d}$ near the $3 p 5 d^{1} F$ and $3 p 7 d^{1} F$ resonances, respectively, compares with the energy-independt effective initial radial function $\xi_{6 d}$. The overlapping integral $O_{\nu \nu_{\mathrm{i}}}=<\xi_{\nu_{\mathrm{i}} d} \mid \xi_{\nu d}>$ changes sign near the resonance due to the quasi-orthogonality between the initial and effective final state radial functions of the outer $d$ electrons (Fang et al, 1997). 

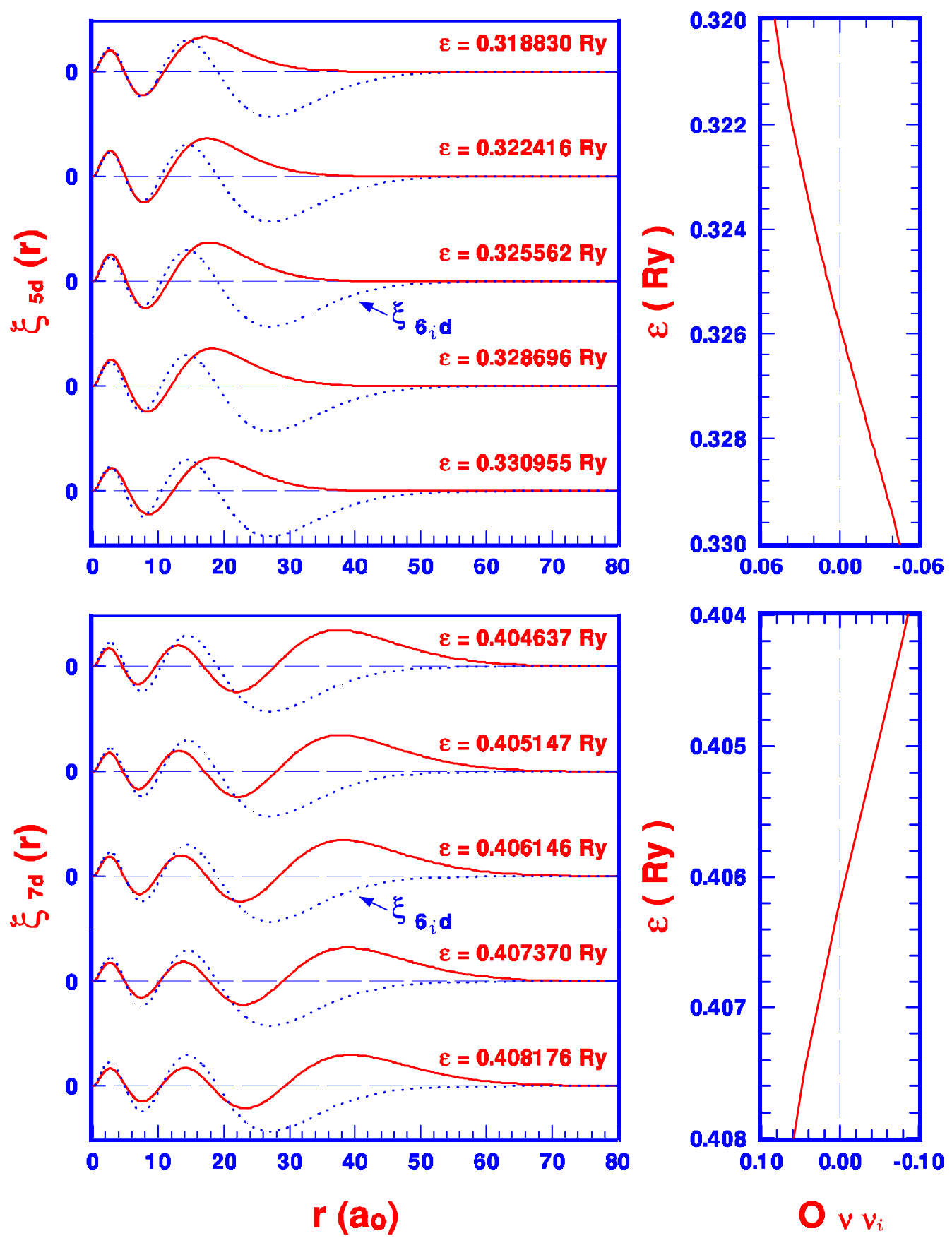

\section{$E_{r}\left(3 p 5 d^{1} F\right)=0.325556 R y \quad E_{r}\left(3 p 7 d^{1} F\right)=0.405490 R y$}

Fig. 8. The $A l^{+} 3 s 6 d^{1} D \rightarrow{ }^{1} F$ photoionization near the $3 p 5 l$ and $3 p 7 l^{1} F$ resonances and the individual contributions $F_{f i}$ to the total transition amplitude $F_{f i}^{t}$ from processes i)-iv) (Fang et al, 1997). 

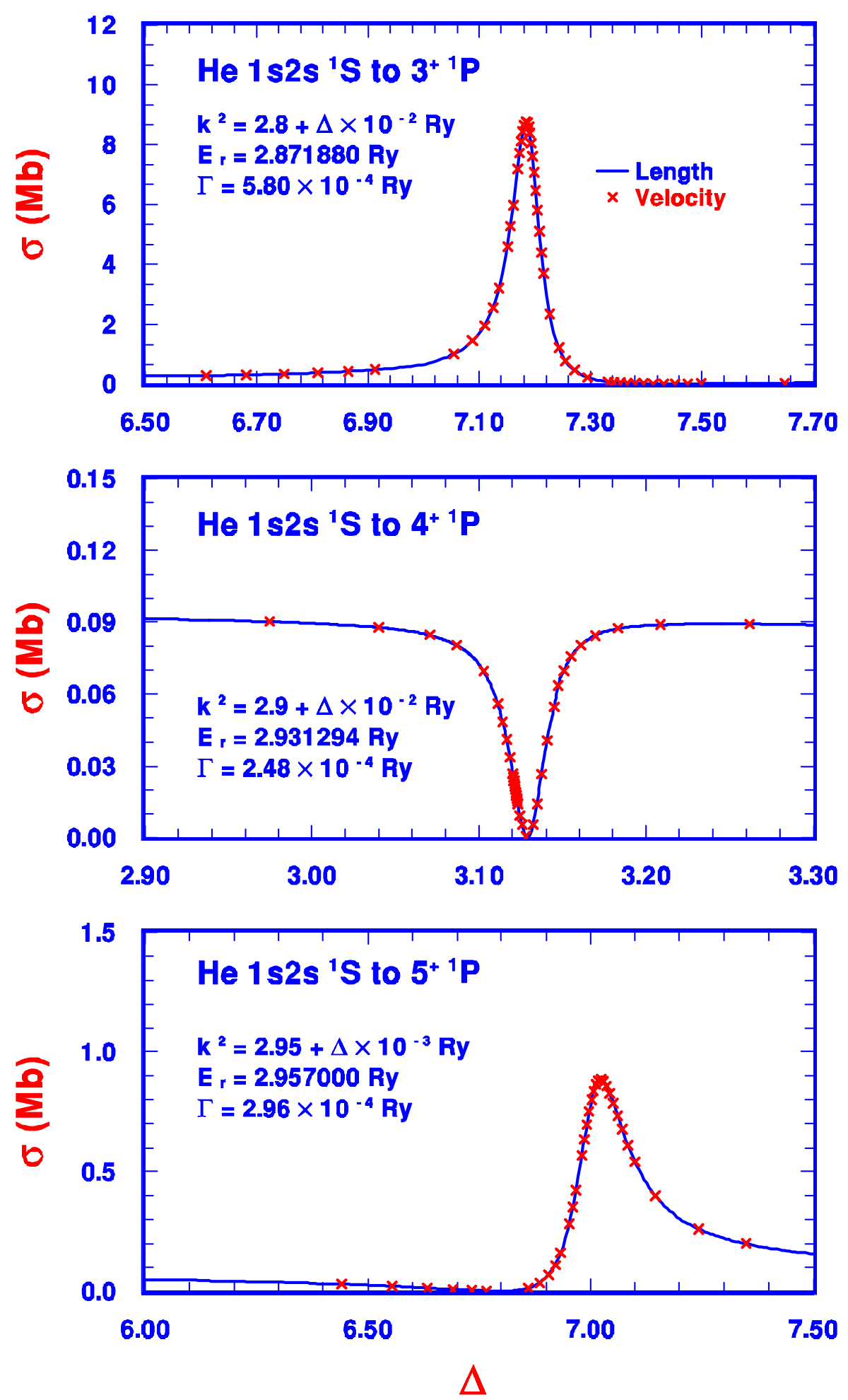

Fig. 9. Variation of the He $1 s 2 s^{1} S \rightarrow{ }^{1} P$ photoionization resonance structures along the $s p, 2 n^{+}$autoionization series (Fang and Chang, 1997). 

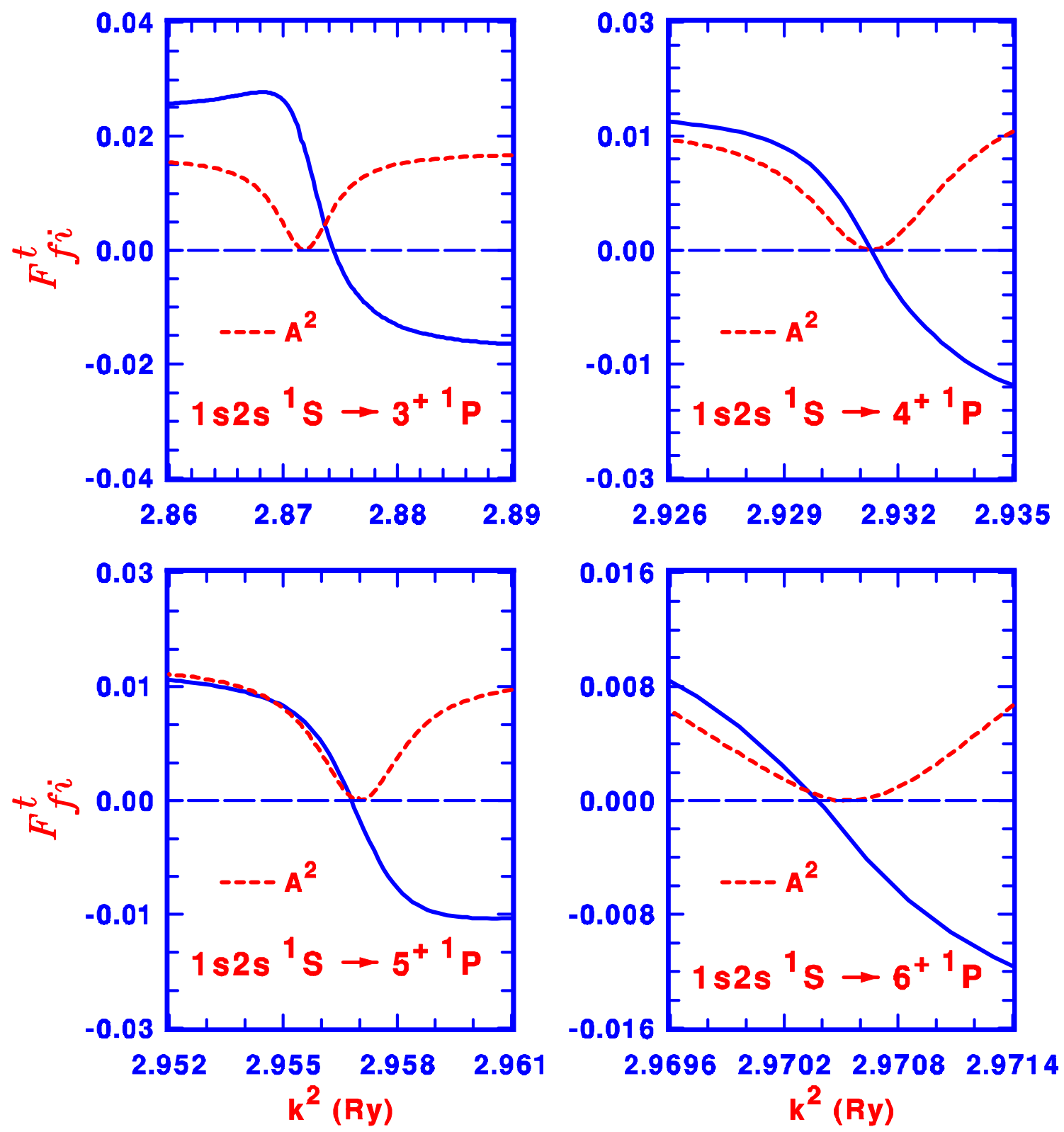

Fig. 10. The energy variation of the total transition amplitudes $F_{f i}^{t}$ (solid curve) and the square of the amplitude $A$ of the oscillating effective oneparticle functions representing the outgoing photoelectron from the $1 s 2 s^{1} S \rightarrow s p, 2 n^{+1} P$ photoionization (Fang and Chang, 1997). 

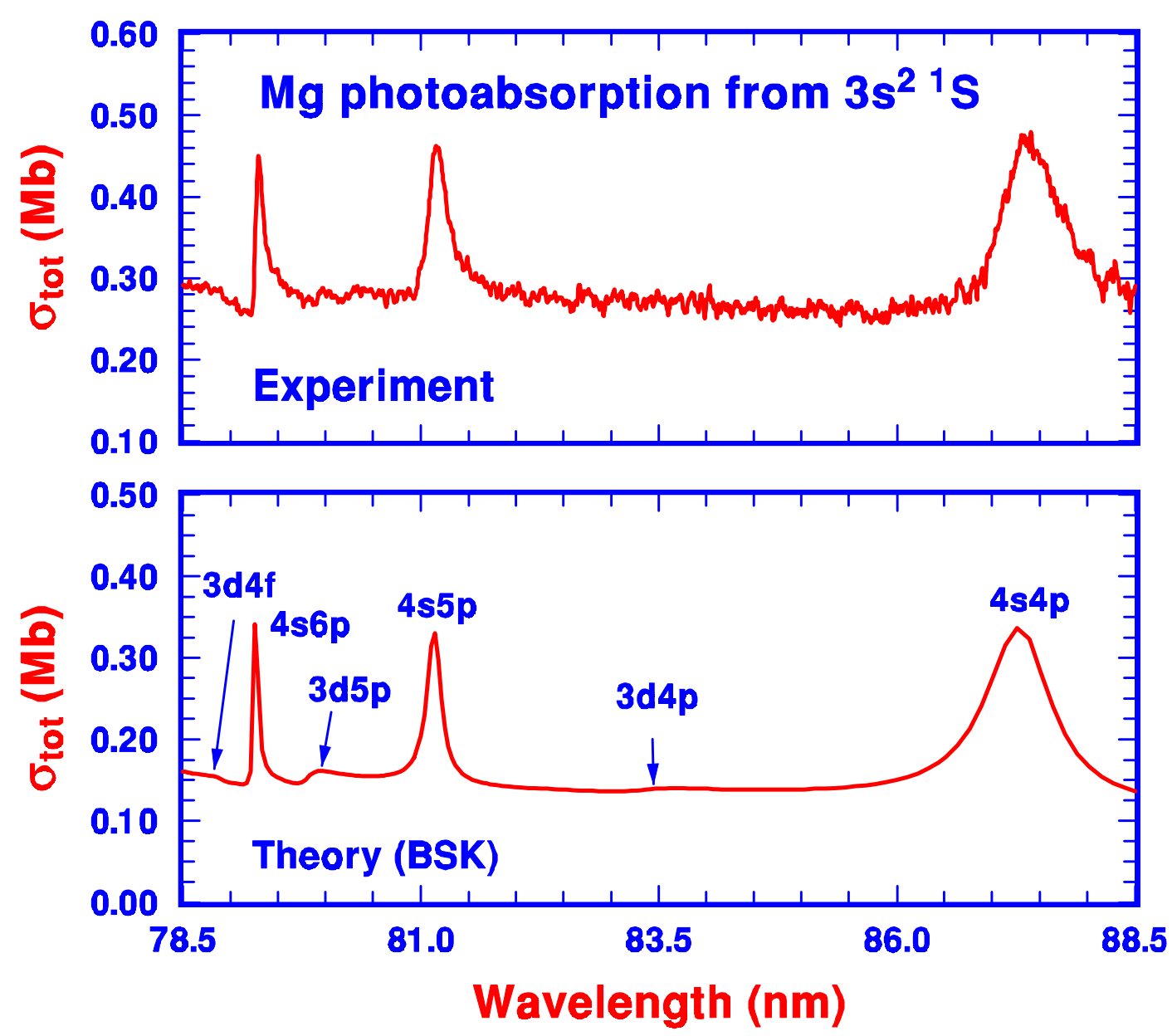

Fig. 11. Comparison between measured absolute Mg photoabsorption cross sections and the theoretical photoionization cross sections using BSK approach from $78.5 \mathrm{~nm}$ to $88.5 \mathrm{~nm}$ (Fung et al, 2001). 

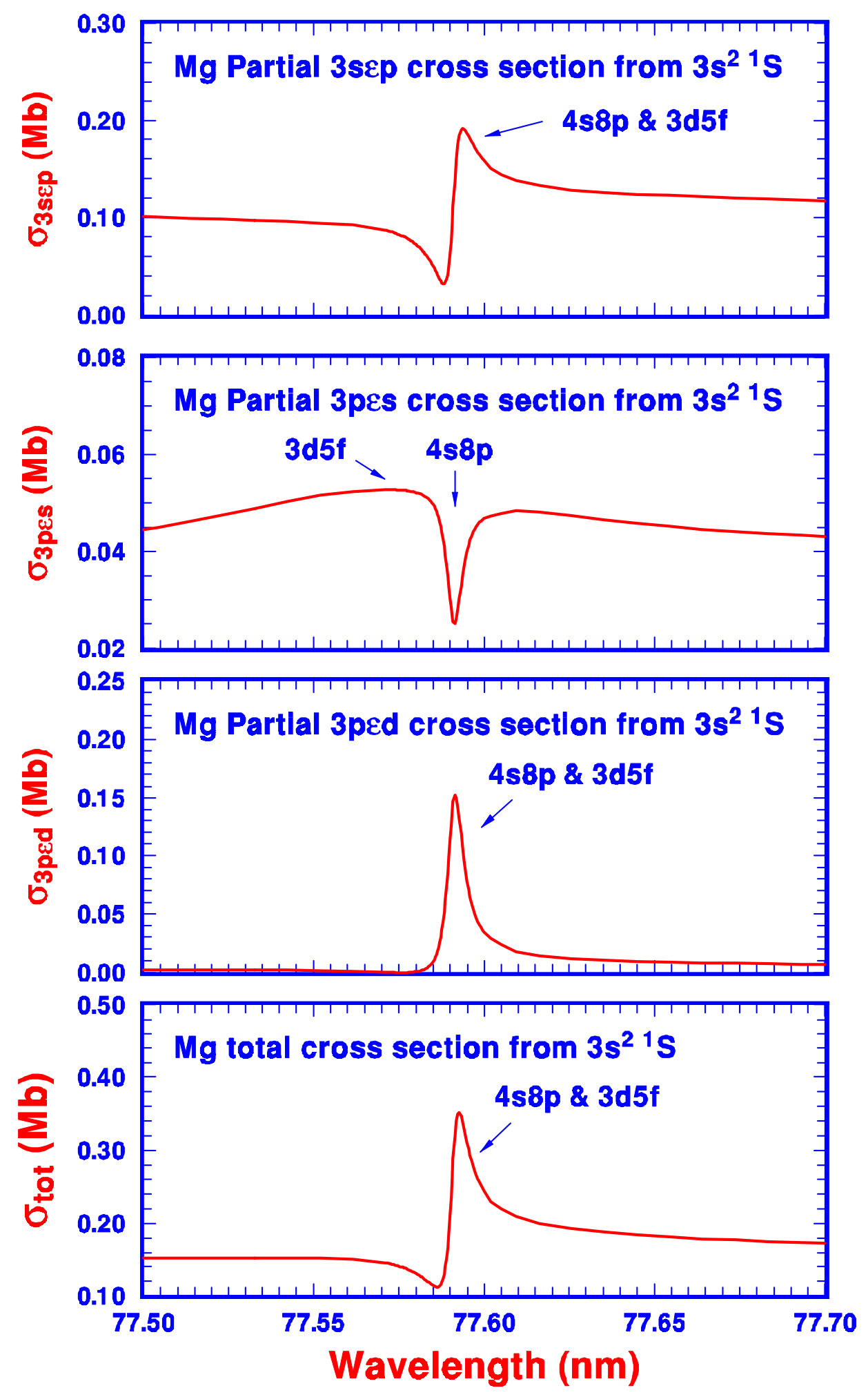

Fig. 12. The overlapping narrow $\mathrm{Mg} 4 s 8 p^{1} P$ resonance and the broad $3 d 5 f^{1} P$ resonance near $77.6 \mathrm{~nm}$ (Fung et al, 2001). 


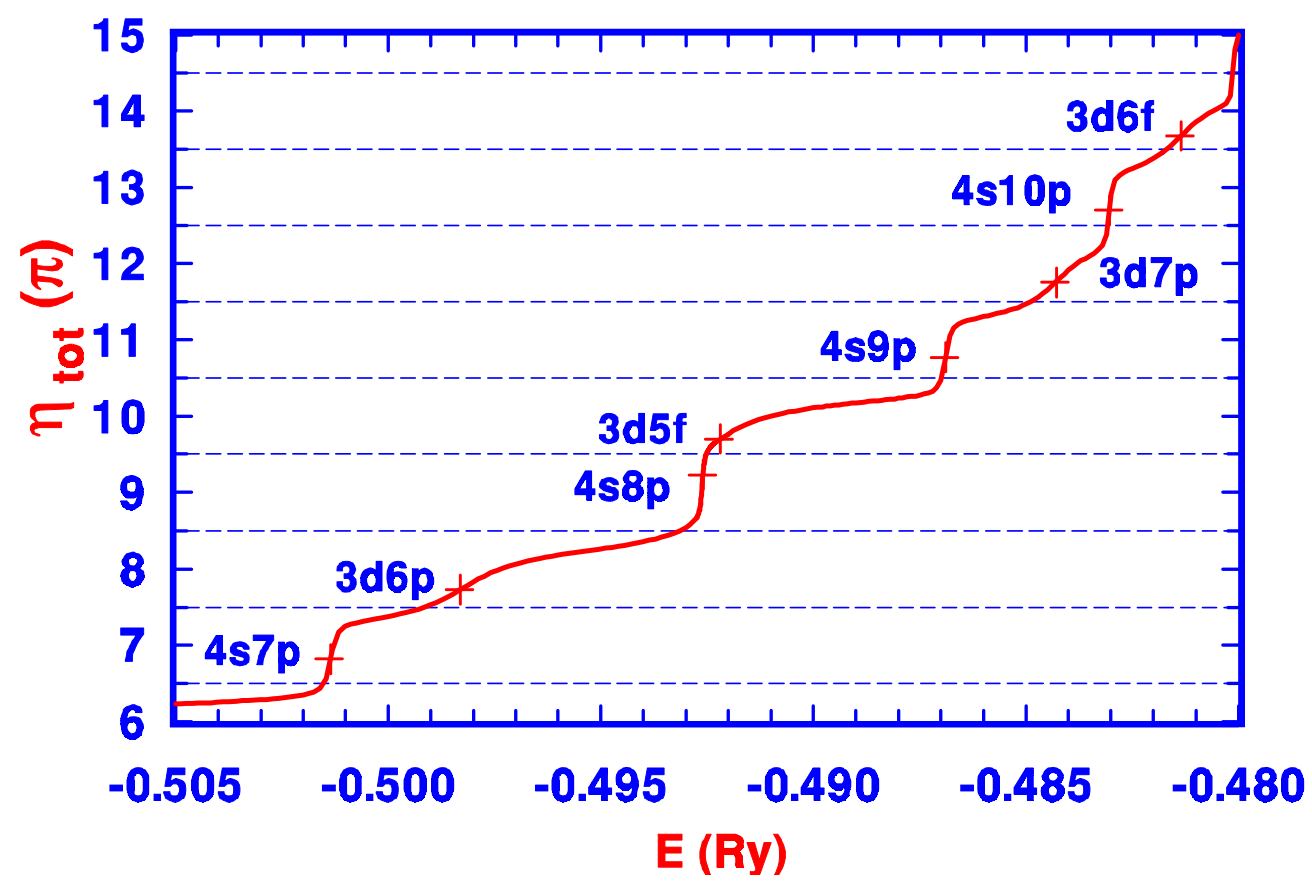

Fig. 13. The energy variation of $\eta_{t o t}$ where $E$ in $R y$ is measured against the $M g^{+2}$ threshold (Fung et al, 2001).
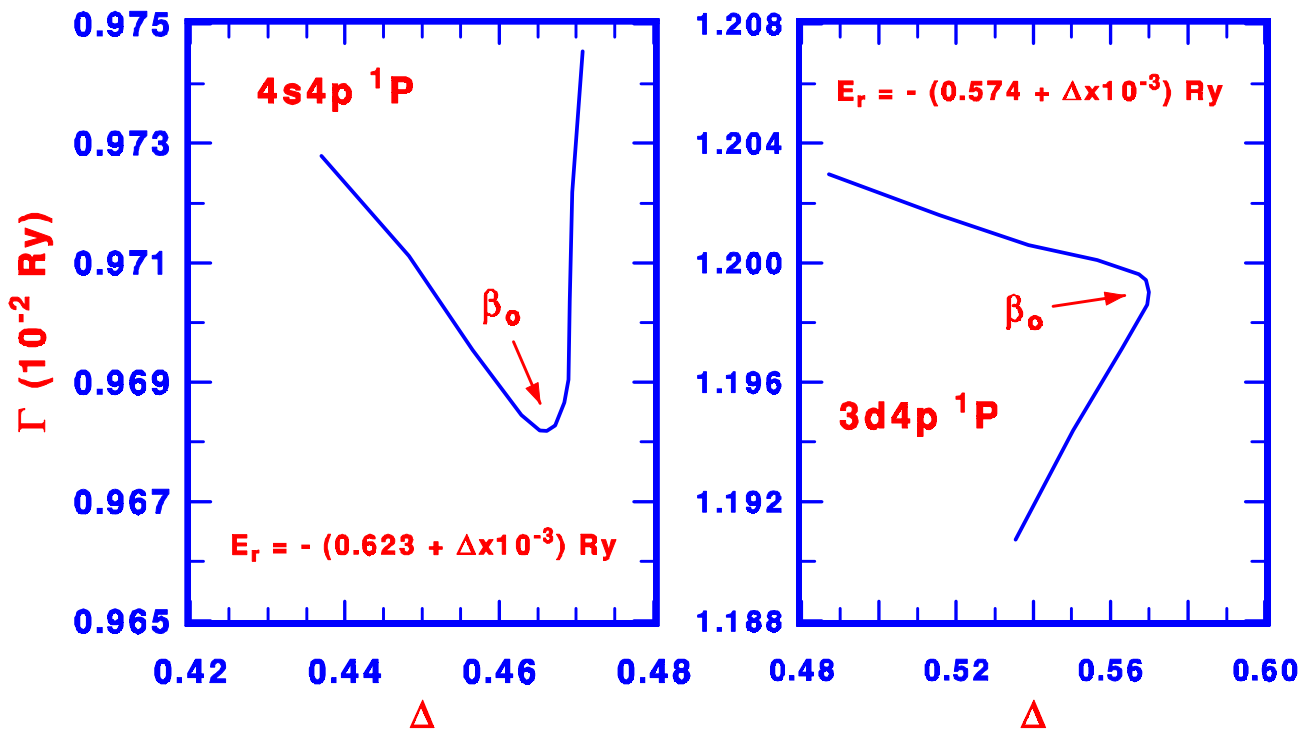

Fig. 14. The variations of $E_{r}$ and $\Gamma$ of the $\operatorname{Mg} 4 s 4 p^{1} P$ and $3 d 4 p^{1} P$ resonances for different $\beta$ values at $\theta=0.4$ radians (Fang et al, 2002). 